Homology, Homotopy and Applications, vol.10(1), 2008, pp.437-479

\title{
ON THE HOMOTOPY GROUPS OF TORIC SPACES
}

\author{
DAVID ALLEN
}

\author{
(communicated by Donald M. Davis)
}

\begin{abstract}
Given a certain class of simple polyhedral complexes $P$ and the associated Borel space $B_{T} P$ we compute the $E_{2}$-term of the Unstable Adams Novikov Spectral Sequence for $B_{T} P$ through a range. As a result, through a range, the higher homotopy groups of $B_{T} P$ are isomorphic to the homotopy groups of a wedge of spheres whose dimensions depend on the combinatorics of $P$. This paper provides a unified approach to attacking the problem of computing the higher homotopy groups of complements of arbitrary complex coordinate subspace arrangements. We extend all higher homotopy group computations in the cases where the homotopy type of a complement of a complex coordinate subspace arrangement is unknown. If $K$ is a simplicial complex that defines a triangulation of a sphere that is dual to a simple convex polytope $P$, then, in many cases, the homotopy groups of the quasi-toric manifold $M^{2 n}(\lambda)$ can be computed through a range that was previously unknown. As an application, the homotopy type of a family of moment angle complexes $Z_{K}$ will be determined.
\end{abstract}

\section{Introduction}

The higher homotopy groups of a quasi-toric manifold and a complex coordinate subspace arrangement complement are related by the existence of various fibrations. Quasi-toric manifolds do not exist in general if $P$ is not dual to a simplicial sphere. However, by the work of [BP4] the higher homotopy groups of $Z_{K}$ can be attacked by studying $\pi_{*}\left(B_{T} Z_{K}\right)$. This paper uses the methods of unstable homotopy theory to understand the homotopy groups of various toric spaces. In particular, a general framework for understanding $\pi_{*}\left(B_{T} P\right), \pi_{*}\left(Z_{K}\right)$ and $\pi_{*}\left(M^{2 n}(\lambda)\right)$ is discussed. Some inroads have been made with respect to the homotopy groups of certain subspace arrangements and their complements by the work of [GT, L, DP, PS], and [Zie]. It turns out that in many cases only the first non-trivial higher homotopy group is known. Imposing mild combinatorial restrictions on the simple polyhedral complex $P_{K}$ dual to a simplicial complex $K$, [DJ, p. 428], we are able to write down the

Received April 24, 2007, revised October 2, 2007; published on June 4, 2008.

2000 Mathematics Subject Classification: Primary 47A15, Secondary 46A32, 47D20.

Key words and phrases: homotopy group, quasi-toric manifold, subspace arrangement complement. This article is available at http://intlpress.com/HHA/v10/n1/a19

Copyright (c) 2008, International Press. Permission to copy for private use granted. 
$E_{2}$-term of the UANSS within a range. However, more stringent restrictions must be imposed on the underlying combinatorial object if one wants to use filtration arguments to determine the homotopy type of $Z_{K}$. In [GT2] it was shown that when $K$ is a shifted complex the homotopy type of $Z_{K}$ is a wedge of spheres. To illustrate the power of the techniques developed in this paper the homotopy type of families of $Z_{K}$ can be determined for certain $K$ which are not shifted complexes. It follows from this analysis and the work of [GT2] that we have constructed families of simplicial complexes that are not shifted but whose face ring is Golod.

We start by computing $B P_{*}\left(B_{T} P\right)$. We generalize the notion of injective extension sequence to injective extension sequence through a range. Injective extension sequences first appeared in [MS] and were used by [Bous] to compute the primitive dimension of co-algebras. The obstruction to complete answers as far as homotopy calculations go is the failure of the existence of injective extension sequences without the range restriction that comes about from the combinatorics. In general, it turns out that the degree of a particular "relation among relations" provides the upper bound for the range for which an injective extension sequence exists. The relationship between the combinatorics and the theory of derived functors of non-additive functors is uncovered. Bousfield [Bous] alludes to the idea of using the higher-derived functors of the primitive element functor, $R^{i} P$, to measure the failure of an algebra being free. It is shown that $R^{1} P$ comes from the relations in the face ring $k(K)$. Even though it is well known that $R^{2} P$ corresponds in some way to a relation among relations, the precise way in which this holds given certain combinatorial restrictions is exposed. From this, the generators of $R^{2} P$ are represented by cycles in a chain complex, $U$, which comes about from applying the primitive element functor to a certain resolution in a non-Alain category.

Since $Z_{K}$ exists for any simplicial complex $K$ we have the associated Borel space $E T^{m} \times_{T^{m}} Z_{K}$. The techniques developed in this paper show that $\pi_{*}\left(B_{T} P\right)$ $\cong \pi_{*}\left(Z_{K}\right) \cong \pi_{*}\left(\bigvee_{i} S^{j}\right)$ within a range, where $P$ is dual to $K$ in some way. The number of factors and the dimensions of the spheres are intimately related to the combinatorics of $P_{K}$. In this context, $\eta$ refers to multiplication by the co-operation $h_{1}$, which is an element of the Hopf algebroid $\Gamma=B P_{*}(B P)$. More specifically, the number of $\eta$ towers that appear in the $E_{2}$-term of the UANSS is indexed by the cardinality of the set of generators of $I-|I|$, where $I$ is the ideal in the face ring generated by square-free monomials that come from either the missing faces of $K$ or the trivial intersections of facets in $P$. As an application, the homotopy type of a family of complex subspace arrangement complements is determined by showing that a certain attaching map is trivial via a filtration argument. Arguments of this sort arise from analyzing the $E_{2}$-term of the UANSS. The geometry and square-freeness of the ideal $I$ in $k(K)$ is critical in determining the stable and unstable co-actions. It is not clear how one would compute these maps without the existence of the map $B_{T} P \rightarrow \prod_{m} \mathbb{C} P^{\infty}$.

The paper is set up as follows. The preliminaries are discussed in $\S 2-\S 5$. In $\S 4$ the tools used in unstable homotopy theory are listed for the benefit of the non-expert. In $\S 5$ injective extension sequences are described as well as the notion of an injective extension sequence through a range which will be crucial in what follows. In $\S 6$ the homotopy groups of $B_{T} X$ are studied. 


\section{Acknowledgements}

The author would like to thank Martin Bendersky and Tony Bahri for their generosity and patience over the years in which this work was completed. The author would also like to thank the referee, whose suggestions improved the paper.

\section{Main results and notational conventions}

In this section, we state the main results and set up the notational conventions that will be assumed throughout the paper.

Let $K$ be an $n$-1-dimensional simplicial complex with the vertex set $\{1, \ldots, m\}$. In short, we say $K$ is a simplicial complex. If it is necessary to refer to the vertex set, then we shall say $K$ is a simplicial complex on $[m]$. If the $n$-dimensional simple polyhedral complex $P$ is obtained as the cone over the barycentric subdivision of $K$, we write $P_{K}$ [DJ]. If $K$ is a simplicial sphere, then $P_{K}$ is a simple convex polytope. Fix $K$ and write $P$ for $P_{K}$. Let $F=\left\{F_{1}, \ldots, F_{m}\right\}$ be the set of facets of $P$ and the number of facets will be denoted by $m$. For a fixed commutative ring $R$ with unit, the face ring of $P$ is

$$
R(P)=R\left[v_{1}, \ldots, v_{m}\right] / I=\left\langle v_{i_{1}} \cdots v_{i_{k}} \mid \bigcap_{j=1}^{k} F_{i_{j}}=\emptyset\right\rangle,
$$

where $\left|v_{i}\right|=2$ are indexed by the facets and the ideal $I$ is generated by square-free monomials $r_{1}, \ldots, r_{k}$. Suppose that the monomials $r_{i}$ that generate the ideal $I$ in the face ring are of degree $\left|r_{i}\right|$. The cardinality $|I|$ of the ideal $I$ is equal to the cardinality of the set of monomials that generate $I$. This set of monomials contains no redundancies. We say that $P$ is $q$-neighborly if the intersection of any $q$ facets is non-empty.

It is assumed throughout that $P$ is $\mathbf{q} \geqslant 1$ neighborly unless stated otherwise. Simplicial complexes $K$ are assumed to be $q$-neighborly as well, [BP4, p. 96]. References to neighborliness will only be made to clarify statements. We call $F=B P_{*}\left(B_{T} P\right)$ the $B P$-face ring. Let $\Re_{\text {min }}$ be a relation among relations of minimal degree (6.2). Many of the theorems that appear in $\S 6$ depend on the neighborliness of $P$, since they hold in a range specified by $\left|\Re_{\text {min }}\right|$ which depends on $q$. In fact, the theorems hold in the range $2 q+4 \leqslant\left|\Re_{\text {min }}\right| \leqslant 4 q+2 k_{i j}+2$, where the $k_{i j}$ are positive integers given by Definition 6.7.

Theorem 6.15. Given a simple polyhedral complex $P$, then up to dimension $\left|\Re_{\text {min }}\right|-1$, the $B P_{*}$-module structure of $R^{i} P F$ is as follows:

$$
R^{i} P F \cong \begin{cases}B P_{*}\left\{a_{1}, \ldots, a_{m}\right\}, & i=0,\left|a_{i}\right|=2 \\ B P_{*}\left\{e_{1} \ldots, e_{k}\right\}, & \text { if } i=1,\left|e_{j}\right|=\left|r_{j}\right| \\ 0 & \text { otherwise. }\end{cases}
$$

Various stable and unstable co-actions are computed through a range. The way in which the geometry and combinatorics reveals itself is interesting when computing these maps. After various identifications we have the following through a range.

Theorem 6.26. The unstable co-action on $R^{1} P F$ is trivial. 
It is assumed throughout that the coefficient ring is $B P_{*}$. Modules are assumed to be positively graded $B P_{*}$-modules which are free of finite type [BCR, p. 378]. In this context, a co-algebra means a co-algebra generated by modules of this type [BCR, p. 378]. It turns out that the co-algebra structure is essential in computing the $G$-co-algebra structure map, $C \rightarrow G(C) 4.2$, where $G$ is the functor of a cotriple on the category of free positively graded $B P_{*}$-modules. Further analysis shows that the square-freeness of the ideal $I$ in the face ring allows the $\Gamma=B P_{*}(B P)$ co-action on various co-algebras to be computed. The category of unstable co-algebras $\mathcal{G}$, consists of free $B P_{*}$-modules equipped with maps: $M \rightarrow G(M), M \rightarrow M \otimes_{B P_{*}} M$ and $M \rightarrow \Gamma \otimes_{B P_{*}} M$ that come from a particular cotriple. The statements of the main theorems on the homotopy groups of $B_{T} P$ concern the notion of a spectral sequence converging within a range. In particular, $[\mathbf{B C M}]$ define a Composite Functor Spectral Sequence (CFSS) that converges to the $E_{2}$-term of the Unstable Adams Novikov Spectral Sequence (UANSS). The classes that appear in the $E_{2}$-term of the UANSS are those that survive to the $E_{\infty}$ in the CFSS. The input for the CFSS consists of the higher-derived functors of the primitives as well as their structure as an unstable comodule in a particular category. The co-action formulae and computation of the higher-derived functors hold within a range, which is determined by the combinatorics of the underlying $P$.

The main theorems concerning the homotopy groups of toric spaces are Theorems 6.33 and 6.35 .

Theorem 6.33. If $K$ is a simplicial complex and $F$ is the $B P_{*}$-face ring associated to $P$, then in filtration $s \geqslant 0$ and total degree $t \leqslant s(2 p-2)+\left|\Re_{\text {min }}\right|-1$. For $i=0,1$, the classes in

$$
\operatorname{Ext}_{\mathcal{G}}^{i+s, t}(F)
$$

are those that come from the $E_{2}$-terms:

$$
\operatorname{Ext}_{A(U)}^{s, t}\left(B P_{*}\left(\prod_{r_{j} \in I} S^{\left|r_{j}\right|}\right)\right) \quad \text { for } i=1
$$

and

$$
\operatorname{Ext}_{A(U)}^{s, t}\left(B P_{*}\left\{a_{1}, \ldots, a_{k}\right\}\right) \quad \text { for } i=0 .
$$

By relabeling the axes in the $E_{2}$ page of the UANSS and using the condition on the total degree that appears in Theorem 6.33 we obtain a restriction on the stem degree in homotopy. We now have the following theorem on $\pi_{*}\left(B_{T} P\right)$.

Theorem 6.35. Given $P$ and total degree $t$ as in Theorem 6.33, let $B_{T} P$ be the associated Borel space. We have

$$
\pi_{t-s-1}\left(B_{T} P\right)_{(p)}=\pi_{t-s-1}\left(\left(\bigvee_{r_{j} \in I} S^{\left|r_{j}\right|-1}\right)\right)_{(p)}
$$

for $t-s \leqslant\left|\Re_{\text {min }}\right|-1$ and in filtration $s=0, \pi_{2}\left(B_{T} P\right)=\mathbb{Z}^{\oplus m}$.

These computations generalize what is known about the homotopy groups of the Borel space and the various toric spaces associated with $P$. One should notice that the 
homotopy groups of $B_{T} P$ are known through a range. If $K$ is a simplicial sphere then this theorem has, by way of the standard fibrations, applications to the homotopy groups of quasi-toric manifolds. There are applications pertaining to the homotopy type of complex coordinate subspace arrangement complements associated to a particular family of simplicial complexes $K_{[m]}$ (Definition 6.41), which are modeled on the complex dual to the square in that each simplicial complex in this family has exactly two missing faces and the monomials that generate the ideal $I$ in the face ring have a " $v$ " in common, Definition 6.41.

Theorem 6.54. Let $m \geqslant 4$ be an integer and $Z_{K_{[m]}}$ the moment angle complex associated to $K_{[m]}$. We have

$$
Z_{K_{[m]}} \simeq \begin{cases}S^{2 r-1} \bigvee S^{2 m-2 r+1} \bigvee S^{2 m-2} & m \text { even } \\ S^{2 r-1} \bigvee S^{2 r-1} \bigvee S^{2 m-2} & m \text { odd }\end{cases}
$$

Recall from [GT2] the definition of a shifted complex. A simplicial complex $K$ is shifted if there is an ordering on the vertex set such that whenever $\sigma \in K$ and $v<v^{\prime}$, then $\sigma \backslash\{v\} \cup v^{\prime} \in K$. Closer inspection of the simplicial complexes $K_{[m]}$ reveals that they are not shifted for certain $m$. For example, when $m=5$, let $\sigma=\{1,2,4,5\}$ $\in K_{[5]}$. However, $\sigma \backslash\{4\} \cup 3 \notin K_{[5]}$ as described in [GT2]. As a result, the techniques in that paper cannot be used to determine the homotopy type of the moment angle complexes associated to various $K_{[m]}$. The techniques used in this paper require the analysis of the attaching maps present in the CW-structure of $Z_{K}$ as certain homotopy classes of maps, which can be studied via spectral sequence arguments.

\section{Quasi-toric manifolds}

Suppose that $P$ is a $n$-dimensional simple convex polytope. Let $\Im$ denote the set of facets of $P$. Given the torus $T^{m}$ the coordinate torus $T_{\iota_{1}, \ldots, \iota_{k}}^{k}$ is a product of tori that come from the factors $\iota_{1}, \ldots, \iota_{k}$ in $T^{m}$.

Definition 2.1. Let $\lambda: \Im \rightarrow \mathbb{Z}^{n}$ be a function which assigns to each coordinate torus a primitive vector in the integer lattice $\mathbb{Z}^{n}$. We call such a function the characteristic function of $M^{2 n}$.

Since $P$ is simple any codimension $k$-face $F$ can be written as the intersection: $F_{k}=\bigcap_{i=1}^{k} F_{i_{j}}$, where $F_{i_{j}} \in \Im$. The $\lambda$ map determines a subgroup $G_{F}$ of the lattice $\mathbb{Z}^{n}$.

It was shown in $[\mathbf{D J}]$ that a quasi-toric manifold can be constructed from a certain quotient space $T^{n} \times P^{n} / \sim$. We will take this as our definition of a quasi-toric manifold. First, one needs a particular pair $\left(P^{n}, \lambda\right)$, where $\lambda$ is a specific linear map defined below. Given an $n$-dimensional simple convex polytope $P$ and a codimension $k$ face, $F$ of $P$, we let $G_{F}$ denote the stabilizer of $F$ under the torus action for any $x \in F$. For any $F \in \Im, G_{F}$ is a rank-one subgroup (coordinate torus). Coordinate tori are determined by primitive vectors in the integer lattice $\mathbb{Z}^{n}$ up to sign. This gives a prescription for defining a function. In the next definition let $n=\operatorname{dim} P$.

Definition 2.2. Let $F_{k}$ be a codimension $k$-face of $P$. We define $\lambda\left(F_{k}\right)$ as

$$
\mathbb{Z}-\operatorname{span}\left\langle\lambda\left(F_{i_{1}}\right), \ldots, \lambda\left(F_{i_{k}}\right)\right\rangle \subset \mathbb{Z}^{n} .
$$


The subgroup $G_{F}$ is determined by the sub-lattice that is generated by the image of $\lambda$ defined above. Since $P$ is simple, any vertex $v \in P$ can be written as the intersection of exactly $n$ facets. This implies that the subspace $\lambda(v)$ is a basis for $\mathbb{Z}^{n}$. If one puts a rather restricted condition on the image of $\lambda$ then it is possible to construct families of quasi-toric manifolds over $P$. Davis and Januszkiewicz $[\mathbf{D J}]$ refer to this restriction as condition $(*)$. Let $F_{k}$ be a codimension $k$-face of $P$. Let $\lambda\left(F_{k}\right)$ be given as above. We say $\lambda$ satisfies condition $(*)$ if $\lambda\left(F_{k}\right)$ spans a $k$-dimensional uni-modular subspace of $\mathbb{Z}^{n}$.

In the general setting we suppose that $K$ is a $(n-1)$-dimensional simplicial complex and let $\lambda: \operatorname{Ver}(K) \rightarrow \mathbb{Z}^{n}$ be a function. For each $(k-1) \operatorname{simplex} \sigma \in K$ let $E_{\sigma}$ be the $\mathbb{Z}$-span of $\lambda(v)$ where $v \in \sigma$. In this context, condition $(*)$ translates as follows: for each $\sigma$ as above, $E_{\sigma}$ is a $k$-dimensional uni-modular subspace of $\mathbb{Z}^{n}[\mathbf{D J}]$. The resulting space is a toric space and not necessarily a quasi-toric manifold. We are ready to describe the construction of quasi-toric manifolds from pairs of the form $(P, \lambda)$ where $\lambda$ satisfies condition $(*)$. Buchstaber and Panov [BP4] call such pairs characteristic pairs. For the convenience of the reader we list the following from [DJ].

Construction 2.3. Consider the characteristic pair $(P, \lambda)$. The following construction will produce a family of quasi-toric manifolds $M^{2 n}(\lambda)$ dependent on $\lambda$ that sit over $P[\mathbf{D J}]$. The relation between this construction and the construction of toric varieties from the normal fan $\Sigma(P)$ associated to $P$ can be found in [BP4, 5.1.3]. Let $F(p)$ be the unique face of $P$ which contains the point $p$ in its interior. Suppose we have an action of the torus on $P$. Define an equivalence relation $\sim$ on $T^{n} \times P^{n}$ by $(g, p) \sim(h, q) \Leftrightarrow p=q$ and $g^{-1} h \in G_{F(p)}$. Denote the resulting quotient space by $M^{2 n}(\lambda)$. It is shown in $[\mathbf{D J}]$ that this space is a quasi-toric manifold.

As $\lambda$ varies so does the manifold that sits over $P$. It has been shown that the homology groups of $M^{2 n}(\lambda)$ are independent of $\lambda$ and, in fact, are a function of the $\vec{h}$ associated to $P$-[DJ]. Later, we will show that the homotopy groups in dimensions greater than two are independent of $\lambda$ leading to many interesting observations.

Examples of quasi-toric manifolds would include following. $\mathbb{C} P^{n}$ is a quasi-toric manifold over $\Delta^{n}$. Buchstaber and Ray [BR2] show that the $2 n$-dimensional manifold $B_{n}$ of all bounded flags in $\mathbb{C}^{n+1}$ is a quasi-toric manifold over $I^{n}$, and that $C P^{n} \sharp C P^{n}$ is a quasi-toric manifold over $\Delta^{1} \times \Delta^{n-1}$ by defining a connect sum operation on the level of the polytopes. Orlik and Raymond $[\mathbf{O R}]$ classified four-dimensional quasi-

toric manifolds that sit over polygons and showed that they are connect sum of the Hirzebruch surface with connect sums of $\mathbb{C} P^{2}$.

\section{Moment angle complexes and the Borel space}

If a space $X$ is endowed with a torus action, then we have

Definition 3.1. The Borel space $B_{T} X$ is the identification space

$$
E T^{m} \times X / \sim=E T^{m} \times_{T^{m}} X,
$$

where the equivalence relation is defined by: $(e, x) \sim\left(e g, g^{-1} x\right)$ for any $e \in E T^{m}$ and $x \in X, g \in T^{m}$. 
Remark 3.2. There is no ambiguity in writing $B_{T} P$ instead of $B_{T} X$, the Borel construction applied to a space. $B_{T} P$ refers to $E T^{m} \times X / \sim$ which has the homotopy type of $P$ when $P$ is a simple convex polytope $[\mathbf{D J}]$.

Sometimes one refers to this as applying the Borel construction to $X$. One nice property of this definition is that it allows for one to move the torus action into the quotient. Another very important characteristic of the Borel space is the existence of the fibration:

$$
X \longrightarrow E T^{m} \times_{T^{m}} X \longrightarrow B T^{m} .
$$

If $K$ is an $(n-1)$-dimensional simplicial complex on [m], then the Borel construction applied to the corresponding moment angle complex gives the space:

$$
B_{T} Z_{K}=E T^{m} \times_{T^{m}} Z_{K}
$$

When $P$ is an $n$-dimensional simple polyhedral complex, we take the following as our definition of the moment angle complex $[\mathbf{B P 1}, \mathbf{D J}]$ :

\section{Definition 3.3.}

$$
Z_{P}=T^{m} \times P / \sim
$$

where $(g, p) \sim(h, q) \Leftrightarrow p=q$ and $g^{-1} h \in G_{F(p)}$

In fact, $Z_{K}$ exists for any $K$. If $K$ is a simplicial sphere then $Z_{K}$ is a manifold. More generally, $Z_{K}$ is a space. It should be noted that the moment angle complex behaves well with respect to products and other operations on the level of the polytope [BP4]. Neil Strickland has a more general formulation of these spaces [BP4]. In [BP4] it was shown that one can associate the space $D J(K)=\bigcup_{\sigma \in K} B T_{\sigma} \subset B T^{m}$ to any $n$-1-dimensional simplicial complex $K$. It was also shown that for each $K$ there is a deformation retraction $B_{T} Z_{K} \rightarrow D J(K)$. Recall that $U(K)$ is the complex coordinate subspace arrangement complement associated to $K$. Buchstaber and Panov [BP4] show that there is an equivariant deformation retraction $U(K) \rightarrow Z_{K}$.

The homotopy groups of the spaces above are related by the following fibrations which always exist when $P$ is a simple convex polytope:

$$
\begin{aligned}
& T^{m-n} i \longrightarrow Z_{P} \longrightarrow>M^{2 n}, \\
& M^{2 n} \longrightarrow B_{T} M^{2 n} \longrightarrow B T^{n},
\end{aligned}
$$

and

$$
Z_{P} \longrightarrow B_{T} Z_{P} \longrightarrow B T^{m}
$$

By the Borel construction on $Z_{K}$ the last fibration always exists and takes the form:

$$
Z_{K} \longrightarrow B_{T} Z_{K} \longrightarrow B T^{m}
$$

\section{Co-triples and derived functors}

In this section we list the most important constructions and definitions from the theory of derived functors and cotriples over a category. The reader can refer 
to $[$ Bous], $[\mathbf{B C M}],[\mathbf{B C R}]$ or $[\mathbf{B H}]$ for more information. We assume that co-algebras are over the ring $B P_{*}$ and $C$ is a category.

Definition 4.1. A cotriple $(G, \delta, \epsilon)$ consists of a covariant functor $G: C \rightarrow C$ and natural transformations $\delta: G \rightarrow G^{2}$ and $\epsilon: G \rightarrow I$ such that the following diagrams commute:

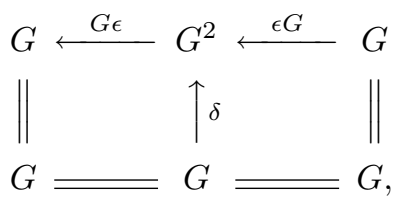

and

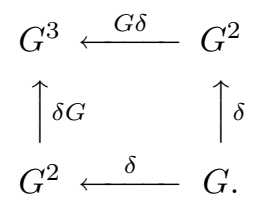

Given a cotriple $G$ we can define a $G$-co-algebra.

Definition 4.2. A $G$-co-algebra is an object $C \in C$ endowed with a map $\psi: C \rightarrow$ $G(C)$ such that the following diagrams commute:

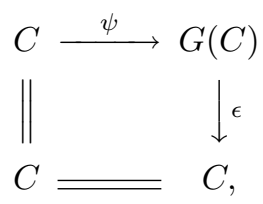

and

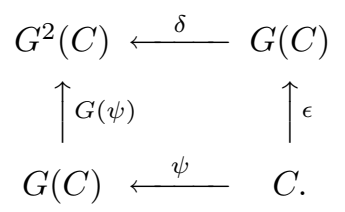

A map of $G$-co-algebras is a map $f: C \rightarrow D$ such that the following diagram commutes:

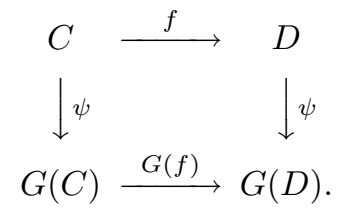

A map of $G$-co-algebras is a map which is compatible with the $G$-co-algebra structure map. The category $M(G)$ consists of pairs $(C, \psi)$, where $C \in C$ and $\psi$ is the $G$-co-algebra structure map. It is important to note that objects of the form $G(C)$ have a canonical $G$-structure defined on them. Specifically, $\delta: G(C) \rightarrow G^{2}(C)$.

Definition 4.3. Let $C \in M(G)$. We call objects of the form $(G(C), \delta)$ the models in $M(G)$. 
Later, we will form unstable resolutions of $G$-co-algebras. We will use the fact that the adjoint of the cotriple $(G, \delta, \epsilon)$ gives rise to a triple $(G, \mu, \eta)$. Specifically, we have the functor $G: C \rightarrow C$ and natural transformations $\mu: G^{2} \rightarrow G$ and $\eta: I \rightarrow G$ such that following diagrams commute:

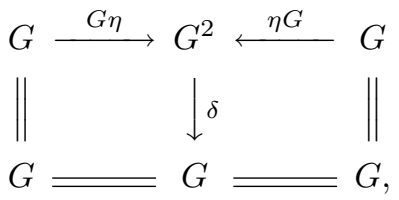

and

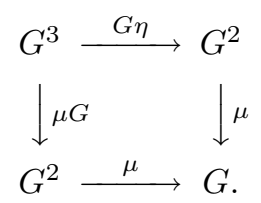

Definition 4.4. Let $D$ be a category. A cosimplicial object $\mathbf{X}$ over $D$ consists of

1. For every integer $n \geqslant 0$ an object $\mathbf{X}^{n} \in D$.

2. For every pair of integers $(i, n)$ with $0 \leqslant i \leqslant n$ coface and codegeneracy maps

$$
\mathbf{X}^{n-1} \longrightarrow d^{i}>>\mathbf{X}^{n}
$$

and

$$
\mathbf{X}^{n+1} \longrightarrow \mathbf{X}^{n}
$$

satisfying the cosimplicial identities:

$$
\begin{aligned}
s^{j} s^{i} & =s^{i-1} s^{j}, i>j, \\
d^{j} d^{i} & =d^{i} d^{j-1}, i<j, \\
s^{j} d^{i} & =d^{i} s^{j-1}, i<j, \\
& =i d, i=j, i=j+1, \\
& =d^{i-1} s^{j}, i>j+1 .
\end{aligned}
$$

Definition 4.5. An augmentation for $\mathbf{X}$ consists of a map $d^{0}: \mathbf{X}^{-1} \rightarrow \mathbf{X}^{0}$ such that the following relation holds:

$$
d^{1} d^{0}=d^{0} d^{0}: \mathbf{X}^{-1} \rightarrow \mathbf{X}^{0} .
$$

Let $A$ be an abelian category, $C O-A$ the category of cosimplicial objects over $A$ and $\mathbf{C h}(\mathrm{A})$ the category of normalized cochain complexes over $A$. We list the Dold-Kan correspondence theorem:

Theorem 4.6. For any abelian category A, there is an equivalence

$$
C O-A \cong \boldsymbol{C h}(A) .
$$

Proof. See [W, p. 271]. 
It follows that cosimplicial objects over a category are in a one to one correspondence with chain complexes over the same category. Let $(G, \delta, \epsilon)$ be a cotriple over $C$.

Definition 4.7. GC is the cosimplicial object over the category $C$ defined by $\mathbf{G} C^{n}=G^{n+1}(C)$.

The cosimplicial object $\mathbf{G} C^{n}=G^{n+1}(C)$ gives rise to a diagram of the form:

$$
\begin{aligned}
C \quad \rightarrow \quad G(C) & \rightarrow \quad G^{2}(C) \\
& \rightarrow \cdots \\
& \rightarrow
\end{aligned}
$$

We call such a diagram the $G$-resolution of $C$. The augmentation $C \rightarrow G(C)$ is just the $G$-co-algebra structure map $\psi: C \rightarrow G(C) . K_{G}$ is a functor from the category $M(G)$ to the category of augmented cosimplicial complexes over $M(G)$. A cosimplicial object can be defined from this functor by $K_{G}(C)^{n}=\mathbf{G} C^{n}$. The structure maps in the $G$-resolution arise by considering the triple $(G, \mu, \eta)$ that comes about from taking the adjoint of the cotriple. The triple structure maps along with the functor $G$ allow for the coface and codegeneracy maps to be defined. We have, $d^{i}=G^{i} \eta G^{n-i}$ and $s^{i}=G^{i} \mu G^{n-i}$.

Let $T$ be a functor: $T: C \rightarrow A$, where $A$ is an abelian category. For $n \geqslant 0$ we apply $T$ to the cosimplicial object GC. Here the category $C$ consists of those objects $C$ with a $G$-co-algebra structure map. $T$ GC is a cosimplicial object over the abelian category $A$. All that is needed to obtain the chain complex that results from applying the functor $T$ to $\mathbf{G C}$ is to define the boundary map.

Definition 4.8. The cochain complex over $A$ associated to the cosimplicial object TGC has boundary map

$$
\partial=\sum_{i \geqslant 0}(-1)^{i} T\left(d^{i}\right)
$$

Let $X$ be a topological space such that $B P_{*}(X)$ is a free $B P_{*}$-module $M$ on the generators $\left\{x_{i}\right\}$. Let $B P(M)$ be the zeroth space of the $\Omega$-spectrum representing the homology theory $B P_{*}(-) \otimes M$.

Definition 4.9. Given $M$ as above we define $G(M)=B P_{*}(B P(X))$.

It is standard to make the following change in notation:

$$
G\left(B P_{*}(X)\right)=G(X)=B P_{*}(B P(X)) .
$$

The category of unstable $G$-co-algebras, $\mathcal{G}$ was first defined in $[\mathbf{B C M}]$. Objects in this category are free $B P_{*}$-modules $M$ equipped with maps: $M \rightarrow G(M)$, $M \rightarrow M \otimes_{B P_{*}} M, M \rightarrow \Gamma \otimes_{B P_{*}} M$ coming from the cotriple structure. Maps between objects are maps that respect the $G$-co-algebra structure.

It is possible to give a space $X$ whose $B P_{*}$-homology satisfies the condition above a $G$-co-algebra structure by applying $B P_{*}(-)$ to the unstable Hurewicz map, $X \rightarrow$ 
$B P(X)$ giving

$$
B P_{*}(X) \rightarrow B P_{*}(B P(X)) .
$$

Recall that $\Gamma=B P_{*}(\underline{B P})$ is the Hopf algebroid of stable co-operations. $\Gamma$ is a bimodule over $B P_{*}$ and comes equipped with the following maps. A product, $\Gamma \otimes_{B P_{*}}$ $\Gamma \rightarrow \Gamma$, left and right unit maps, $\eta_{r}, \eta_{L}: B P_{*} \rightarrow \Gamma$, a diagonal $\Gamma \rightarrow \Gamma \otimes_{B P_{*}} \Gamma$ and a canonical anti-isomorphism, $c: \Gamma \rightarrow \Gamma$ that takes the left action to the right action. The stable co-operation $h_{i}$ is in $\Gamma_{2\left(p^{i}-1\right)}$. $B P$ is a spectrum with two products. First there is $\underline{B P}_{k} \times \underline{B P}_{k} \rightarrow \underline{B P}_{k}$ induced by loop multiplication which induces a $*$ product $B P_{*}\left(\underline{B P}_{k}\right) \otimes_{B P_{*}} B P_{*}\left(\underline{B P}_{k}\right) \rightarrow B P_{*}\left(\underline{B P}_{k}\right)$. Second, there is a map $\underline{B P} \underline{P}_{k} \times \underline{B P}_{r}$ $\rightarrow \underline{B P}_{k+r}$ coming from the ring spectrum structure which induces a circle product $\circ: B P_{*}\left(\underline{B P}_{k}\right) \otimes_{B P_{*}} B P_{*}\left(\underline{B P}_{r}\right) \rightarrow B P_{*}\left(\underline{B P}_{k+r}\right)$. For a multi-index of non-negative integers $I=\left(\iota_{1}, \ldots, \iota_{k}\right), h^{I}=h_{1}^{\circ \iota_{1}} \circ \cdots \circ h_{k}^{\circ \iota_{k}} \in B P_{*}\left(\underline{B P}_{2|I|}\right)$.

The Primitive element functor $P$ is a functor from the category of unstable $G$-coalgebras to the category of positively graded $B P_{*}$-modules free or not.

Definition 4.10. For $M \in \mathcal{G}$ we define $U(M)=(P \circ G)(M)$.

The module $U(M)$ can be determined explicitly using the Ravenel-Wilson basis. In fact, using the non-canonical description of $G(M)=B P_{*}\left(\prod \underline{B P} \underline{P}_{k}\right)$, it was shown by $[\mathbf{R W}]$ that $P B P_{*}\left(\underline{B P}_{k}\right)$ is generated as a Hopf ring by the classes $\left[v^{I}\right] \circ h^{J}$ with certain condition on the multi-indices. Given a $G$ resolution one can apply the functor $P$ to obtain a cochain complex by taking the alternating sums of the coface maps to obtain a particular $U$ complex. $U$ is a functor of a cotriple on the category of positively graded free $B P_{*}$-modules $[\mathbf{B C M}]$. The category of unstable $\Gamma$-comodules, $A(U)$ consists of modules $M$ with maps $M \rightarrow U(M), M \rightarrow \Gamma \otimes_{B P_{*}} M$ coming from the cotriple structure. Maps between objects are maps that respect the $U$-comodule structure.

Proposition 4.11. If $M$ is a free left $B P_{*}$-module, then

1. $U(M)=B P_{*}\left\{h^{I} \otimes m|2 l(I)<| m \mid\right\} \subset \Gamma \otimes_{B P_{*}} M$.

2. If $M$ is an unstable $\Gamma$-comodule, free as a $B P_{*}$-module with co-action $\psi: M \rightarrow$ $U(M)$, then the unstable cobar complex is the chain complex $C^{s, t}(M)=U^{s}(M)_{t}$ with differential given by

$$
\begin{aligned}
d\left(\left[\gamma_{1}\left|\gamma_{2}\right| \cdots \mid \gamma_{s}\right] m\right)= & {\left[1\left|\gamma_{1}\right| \cdots \mid \gamma_{s}\right] m } \\
& +\sum_{j=1}^{s}(-1)^{j}\left[\gamma_{1}|\cdots| \gamma_{j}^{\prime}\left|\gamma_{j}^{\prime \prime}\right| \cdots \mid \gamma_{s}\right] m \\
& +(-1)^{s+1} \sum\left[\gamma_{1}|\cdots| \gamma_{s} \mid \gamma^{\prime}\right] m^{\prime \prime}
\end{aligned}
$$

where $\gamma_{j} \in \Gamma, \psi\left(\gamma_{j}\right)=\sum \gamma_{j}^{\prime} \otimes \gamma_{j}^{\prime \prime}$ and $\psi(m)=\sum \gamma^{\prime} \otimes m^{\prime \prime}$.

Proof. See [BT, pp. 15, 16].

Since $A(U)$ is an abelian category, $\operatorname{Ext}^{s}\left(B P_{*}\left(S^{t}\right),-\right)$ can be computed as the homology of the unstable cobar complex described above. We can now define the right $G$-derived functors of the functor $T$ with respect to objects in $C$. 
Definition 4.12. The right $G$-derived functors of $T$ of a $G$-co-algebra $C$ are defined by:

$$
R^{i} T_{G}(C)=H^{i}(T \mathbf{G C}) .
$$

As an example we will consider $R^{i} P_{G}(M)$, where $M \in S, S$ is the category of coassociative, co-commutative, cofree co-algebras without co-unit over $B P_{*}$, and $P$ is the primitives functor. A more detailed description can be found in $[\mathbf{B C M}]$ and $[\mathbf{B D M}]$.

\section{Injective extension sequences}

In an attempt to keep the notation stable we adopt the following from [BCR]. Most of the details of this section can be found there. Once again, let $S$ denote the category of co-associative, co-commutative, cofree co-algebras without co-unit over $B P_{*}$. A co-algebra refers to a homology co-algebra [Bous].

Definition 5.1. An injective extension sequence is a sequence of maps in $S$.

$$
C^{\prime} \stackrel{f}{\longrightarrow} C \stackrel{g}{\longrightarrow} C^{\prime \prime}
$$

such that

1. $g$ is an epimorphism,

2. $f$ is an inclusion,

3. $C$ is injective as a $C^{\prime \prime}$-comodule,

4. $C^{\prime}=C \square_{C^{\prime \prime}} B P_{*}$.

In other words

$$
C^{\prime} \stackrel{f}{\longrightarrow} \bar{C} \stackrel{\bar{f}}{\longrightarrow} \bar{C}^{\prime}
$$

is a short exact sequence in the category $S$. Recall that modules are assumed to be positively graded $B P_{*}$-modules which are free of finite type. Under this finite generation condition, the functor $\operatorname{Hom}_{B P_{*}}(-,-)$ allows for injective extension sequences to be dualized to projective extension sequences.

Definition 5.2. A projective extension sequence is a sequence of maps in $S^{*}$

$$
\overline{C^{\prime \prime}} \stackrel{\bar{g}}{\longrightarrow} \bar{C} \stackrel{\bar{f}}{\longrightarrow} \bar{C}^{\prime}
$$

such that

1. $\bar{g}$ is an injection,

2. $\bar{f}$ is a surjection,

3. $\bar{C}$ is projective as a $\overline{C^{\prime \prime}}$-module,

4. $\overline{C^{\prime}}=\bar{C} \otimes_{\overline{C^{\prime \prime}}} B P_{*}$.

In particular, a sequence is an injective extension sequence if and only if it is dual to a projective extension sequence. Let $A$ and $B$ be free $B P_{*}$-algebras on evendimensional generators. More specifically, suppose $A=B P_{*}\left[a_{1}, \ldots, a_{k}\right]$, where $\left|a_{i}\right|$ is even and $B=B P_{*}\left[b_{1}, \ldots, b_{r}\right]$ such that $\left|b_{j}\right|$ is even. Suppose $B$ is an $A$ module via a map $f: A \rightarrow B$. 
Definition 5.3. Suppose $i \neq j$. Let $I$ and $I^{\prime}$ be two multi-indexes such that $I \neq I^{\prime}$ and $I, I^{\prime} \subset S=\left\{\iota_{1}, \ldots, \iota_{m}\right\}$. We call a relation of the form:

$$
f\left(a_{i}\right) \prod_{k \in I} b_{k}-f\left(a_{j}\right) \prod_{k^{\prime} \in I^{\prime}} b_{k^{\prime}}=0
$$

in $B P_{*}\left[b_{1}, \ldots, b_{r}\right]$ a relation among relations. We denote such a relation by $\bar{R}$.

Definition 5.4. Let $A$ and $B$ be $B P_{*}$-algebras. The range where $A$ is a free $B$-module is the range where no relations among relations occur.

A strict upper bound on the range for which $A$ is a free $B$-module is given by $|\alpha|$, where $\alpha$ is a relation among relations of minimal degree.

Remark 5.5. The theorems that follow hold in the range where $\bar{C}$ is projective as a $\overline{C^{\prime \prime}}$-module, or, dually, where $C$ is injective as a $C^{\prime \prime}$-comodule.

Injective extension sequences give rise to long exact sequences of higher-derived functors.

Theorem 5.6. Let

$$
C^{\prime} \stackrel{f}{\longrightarrow} C \stackrel{g}{\longrightarrow} C^{\prime \prime}
$$

be an injective extension sequence in $S$. There is a long exact sequence of abelian groups:

$$
0 \longrightarrow P\left(C^{\prime}\right) \longrightarrow P(C) \longrightarrow P\left(C^{\prime}\right) \longrightarrow R^{i} P\left(C^{i}\right) \longrightarrow R^{i} P(C) \longrightarrow R^{i} P\left(C^{\prime \prime}\right) \longrightarrow \cdots
$$

Proof. See $[$ BCR].

Another useful property of injective extension sequences is the fact that they give rise to a long exact sequence of Ext-terms in the category $\mathcal{G}$.

Theorem 5.7. Let

$$
C^{\prime} \stackrel{f}{\longrightarrow} C \stackrel{g}{\longrightarrow} C^{\prime \prime}
$$

be an injective extension sequence in $S$. There is a long exact sequence of Ext-terms

$$
\cdots \operatorname{Ext}_{\mathcal{G}}^{s, t}\left(C^{\prime}\right) \stackrel{f_{*}}{\longrightarrow} \operatorname{Ext}_{\mathcal{G}}^{s, t}(C) \stackrel{g_{*}}{\longrightarrow} \operatorname{Ext}_{\mathcal{G}}^{s, t}\left(C^{\prime \prime}\right) \stackrel{\delta}{\longrightarrow} \cdots,
$$

where the differential $\delta$ has bi-degree $=(1,0)$.

Proof. See $[\mathbf{B C R}]$

The primitives of a co-algebra $M$ are dual to the indecomposables in the dual algebra $F$. The indecomposables lie in a quotient of $F$ and are isomorphic to the module of algebra generators. We have the following

Proposition 5.8. A co-algebra $M$ is cofree if and only if $R^{i} P M=0$ for $i \geqslant 1$ and $M$ is nice if $R^{i} P M=0$ for $i>1$.

Proof. [Bous, p. 479]. 
Examples of nice co-algebras would include $B P_{*}\left(\Omega S^{2 n+1}\right)$ [Bend], as well as $B P_{*}\left(\widehat{S}^{2 n}\right)$, where $\widehat{S}^{2 n}$ is the Toda sphere [BCR]. In the case that $M$ is cofree as a co-algebra we have the following

Theorem 5.9. Let $M \in \mathcal{G}$ and suppose that $M$ is cofree as a co-algebra; then

$$
\operatorname{Ext}_{\mathcal{G}}^{s, t}(M) \cong \operatorname{Ext}_{A(U)}^{s, t}(P M) .
$$

Proof. See $[\mathbf{B C R}]$.

The following result is also proven in $[\mathbf{B C R}]$.

Theorem 5.10. Let $M \in \mathcal{G}$ and suppose that $M$ is nice. Then there is a long exact sequence of Ext-terms:

$$
\cdots \operatorname{Ext}_{A(U)}^{s, t}(P M) \longrightarrow \operatorname{Ext}_{\mathcal{G}}^{s, t}(M) \longrightarrow \operatorname{Ext}_{A(U)}^{s, t}\left(R^{1} P(M)\right) \stackrel{\partial}{\longrightarrow} \cdots,
$$

where $\partial$ has bi-degree $(2,0)$.

In the case that $M$ is not nice as a co-algebra, then the long exact sequence is replaced by a composite functor spectral sequence.

Theorem 5.11. For each $M \in \mathcal{G}$ there is a Composite Functor Spectral Sequence (CFSS) and an UANSS such that the following holds:

$$
\operatorname{Ext}_{A(U)}^{r}\left(B P_{*}\left(S^{t}\right), R_{G}^{s} P(M)\right) \Rightarrow \operatorname{Ext}_{\mathcal{G}}^{r+s}\left(B P_{*}\left(S^{t}\right), B P_{*}(M)\right) .
$$

Proof. See $[\mathbf{B C M}]$.

\section{Homotopy groups of toric spaces}

In this section, the $E_{2}$-term of the UANSS is set up through a range.

\section{Generalities}

$B P_{*}(-)$ denotes unreduced $B P$-homology theory. We recall some facts and notation: $\Gamma$ is the $B P$-analogue of the dual of the Steenrod algebra. Let $K$ be an $(n-1)$ dimensional, $q$-neighborly simplicial complex on $[m]$ and $P_{K}$ the $n$-dimensional $q \geqslant 1$ neighborly simple polyhedral complex which is dual to $K$. If $K$ is a simplicial sphere, then $P_{K}$ is a simple convex polytope. Fix $K$ and write $P$ for $P_{K}$. Throughout this section, $m$ will denote the number of facets of $P$. We also assume that $p$ is a prime number. Recall that $k(K)$ is the algebra

$$
\mathbb{Z}\left[v_{1}, \ldots, v_{m}\right] /\langle I\rangle,
$$

where the $v_{i}$ are dual to the facets in $P,\left|v_{i}\right|=2$ and $\langle I\rangle$ is generated by squarefree monomials $r_{i}$ of degree $\left|r_{i}\right|$. In what follows $B_{T} P$ will denote the Borel space associated to $P$.

Proposition 6.1. Let $E^{*}(-)$ be any complex orientable theory. If $K$ is a simplicial complex, then

$$
E^{*}\left(B_{T} P\right) \cong E_{*}\left[v_{1}, \ldots, v_{m}\right] /\langle I\rangle .
$$


Proof. In the proof of Theorem 4.8 in [DJ, p. 436], it suffices to work with a simplex $\sigma \in K$. Davis and Januszkiewicz $[\mathbf{D J}]$ show that there is a disk bundle $D^{2 k} \rightarrow B_{T} P_{\partial \sigma} \rightarrow B T^{k}$, where $P_{\sigma}$ is the simple polyhedral complex dual to $\sigma$. There is also an associated sphere bundle, $S^{2 k-1} \rightarrow B_{T} P_{\partial \sigma} \rightarrow B T^{k}$. Since $E^{*}(-)$ is complex orientable, there are generalized Chern classes $c_{i}^{E}[\mathbf{A}]$. It was shown in $[\mathbf{D J}]$ that the generators of $H^{*}\left(B T^{k}\right)$ are the first Chern class on the line bundle induced from the diagonal torus action. It is well known that $E^{*}\left(\prod_{k} \mathbb{C} P^{\infty}\right) \cong E_{*}\left[v_{1}, \ldots, v_{k}\right]$ via an Atiyah-Hirzebruch spectral sequence argument $[\mathbf{A}]$. By $[\mathbf{S}$, p. 355], there is an $E$ version of the Gysin sequence for the spherical fibration $S^{2 k-1} \rightarrow B_{T} P_{\partial \sigma} \rightarrow B T^{k}$. As a result, there is an $E^{*}(-)$-analogue to Lemma 4.9 in [DJ, p. 436]. The proof follows by induction on the dimension of $K$, as stated in [DJ].

We will call the algebra $E^{*}\left(B_{T} P\right) \cong E_{*}\left[v_{1}, \ldots, v_{m}\right] /\langle I\rangle$ the $E$-face ring. For the calculations in this paper we will work with the $B P$-face ring. Resolve the algebra in the category of $B P_{*}$-algebras to give the following exact sequence where $\left|x_{j}\right|=\left|r_{j}\right|$,

$$
B P^{*}\left[x_{1}, \ldots, x_{k}\right] \stackrel{\iota^{*}}{\longrightarrow} B P^{*}\left[v_{1}, \ldots, v_{m}\right] \longrightarrow B P^{*}\left(B_{T} P\right)
$$

where $\iota^{*}\left(x_{i}\right)=r_{i}$.

We will use a more specialized version of 5.3. In this case $f=\iota^{*}$, where $\iota^{*}$ is the inclusion, one obtains by resolving the ideal $I$ in the category of free $B P_{*}$-algebras.

Definition 6.2. Suppose $i \neq j$. Let $I$ and $I^{\prime}$ be two multi-indexes such that $I \neq I^{\prime}$ and $I, I^{\prime} \subset S=\left\{\iota_{1}, \ldots, \iota_{m}\right\}$. We call a relation of the form:

$$
\iota^{*}\left(x_{i}\right) \prod_{k \in I} v_{k}-\iota^{*}\left(x_{j}\right) \prod_{k^{\prime} \in I^{\prime}} v_{k^{\prime}}=0
$$

in $B P^{*}\left[v_{1}, \ldots, v_{m}\right]$ a relation among relations. We denote such a relation by $\bar{R}$.

Example 6.3. Consider the following two-dimensional simple convex polytope:

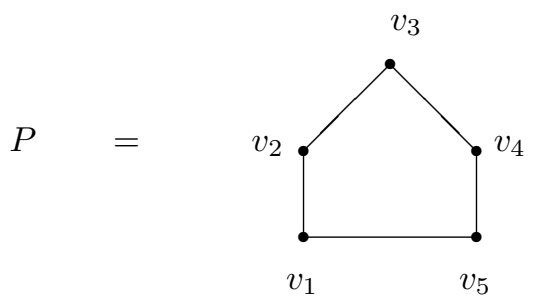

The $B P$-face ring is

$$
F^{*} \cong B P_{*}\left[v_{1}, \ldots, v_{5}\right] / I=\left\langle v_{1} v_{3}, v_{2} v_{4}, v_{3} v_{5}, v_{4} v_{1}, v_{5} v_{2}\right\rangle
$$

The resolution above is

$$
B P^{*}\left[x_{1}, \ldots, x_{5}\right] \stackrel{\iota^{*}}{\longrightarrow} B P^{*}\left[v_{1}, \ldots, v_{5}\right] \longrightarrow B P^{*}\left(B_{T} P\right),
$$


where

$$
\begin{aligned}
& \iota^{*}\left(x_{1}\right)=v_{1} v_{3}, \\
& \iota^{*}\left(x_{2}\right)=v_{2} v_{4}, \\
& \iota^{*}\left(x_{3}\right)=v_{3} v_{5}, \\
& \iota^{*}\left(x_{4}\right)=v_{4} v_{1}, \\
& \iota^{*}\left(x_{5}\right)=v_{5} v_{2} .
\end{aligned}
$$

A relation among relations is $\bar{R}=\iota^{*}\left(x_{1}\right) v_{2} v_{4}-\iota^{*}\left(x_{2}\right) v_{1} v_{3}$. Clearly, $|\bar{R}|=8$.

Definition 6.4. Let $\Re$ denote the set of all possible relations among relations. Define $\Re_{\text {min }}$ to be the relation among relations such that $\left|\Re_{\text {min }}\right| \leqslant|r| \forall r \in \Re$.

$\Re_{\text {min }}$ is a relation among relations of the smallest degree. In general, $\Re_{m i n}$ is not unique.

Example 6.5. Given $F^{*}$ from the previous example, pick $x_{1}$ and $x_{3}$. A relation among relations $\Re_{\min }$ is:

$$
\iota^{*}\left(x_{1}\right) v_{5}-\iota^{*}\left(x_{3}\right) v_{1},
$$

and it follows that

$$
\left|\Re_{\text {min }}\right|=6 .
$$

When given a specific simplicial complex $K$ and an ideal $I$ explicitly, the degree of the smallest relation among relations can be determined. To assess the power of the homotopy calculations that follow we need a more explicit relation between $\left|\Re_{\text {min }}\right|$ and $q$. We need to introduce some notation.

Definition 6.6. For any square-free monomial $m^{\prime} \in B P^{*}\left[v_{1}, \ldots, v_{m}\right]$ we enumerate the number of $v$ 's that appear in $m^{\prime}$ and call it $l\left(m^{\prime}\right)$.

We continue the bookkeeping by introducing the notion of the difference of the lengths of relations.

Definition 6.7. Suppose $r_{i}, r_{j} \in I$ such that $\left|r_{j}\right| \geqslant\left|r_{i}\right|$. Let $k_{i j}=l\left(r_{j}\right)-l\left(r_{i}\right)$.

Proposition 6.8. Given $P$, and $r_{i}, r_{j} \in I$ of minimal degree such that $\left|r_{j}\right| \geqslant\left|r_{i}\right|$, we have $2 q+4 \leqslant\left|\Re_{\text {min }}\right| \leqslant 4 q+2 k_{i j}+2$.

Proof. For $i \neq j$, pick two relations $r_{i}, r_{j} \in I$ of minimal degree such that the $\operatorname{gcd}\left(r_{i}, r_{j}\right) \neq 1$. This implies that the monomials $r_{i}$ and $r_{j}$ have a redundant vertex. There are two cases to consider. First, suppose $\left|r_{j}\right|>\left|r_{i}\right|$. By Definition 6.2, $\left|\Re_{\text {min }}\right|=2 q+2+d$, where $2 q+2$ is the degree of $r_{i}$ and $d$ is the degree of the product of $v_{j}$ 's that appears in Definition 6.2. In the case $\left|r_{j}\right|=\left|r_{i}\right|$ a similar argument gives the desired expression for $\left|\Re_{\min }\right|$. We need a more convenient expression for $d$ and we make the following definition: let $d=2 l\left(\frac{r_{j}}{\operatorname{gcd}\left(r_{i}, r_{j}\right)}\right)$. This integer is the degree of $\prod v_{j}$ that appear in Definition 6.2. To obtain the upper and lower bounds we consider the two extreme cases for the $\operatorname{gcd}\left(r_{i}, r_{j}\right)$. First, suppose $r_{i}$ and $r_{j}$ differ by at most one vertex. This implies $d=2$ from which it is easily deduced that $\left|\Re_{\min }\right|=2 q+4$. 
Notice that this condition on the $g c d$ forces $\left|r_{j}\right|=\left|r_{i}\right|$. In the second case suppose that the relations have only one $v$ in common, a redundant vertex. We determine that $\operatorname{gcd}\left(r_{i}, r_{j}\right)=v$. We claim that $d=2\left(l\left(r_{j}\right)-1\right)$. To see this we recall that $r_{j}$ is a certain monomial and $\frac{r_{j}}{v}$ produces a monomial whose length is $l\left(r_{j}\right)-1$. By the minimality of $\left|r_{i}\right|$ we observe that $l\left(r_{i}\right)=q+1$, hence, by Definition $6.7, l\left(r_{j}\right)=k_{i j}+q+1$. This implies $d=2 k_{i j}+2 q$. However, $\left|\Re_{\text {min }}\right|=2 q+2+d$. The result follows.

$B P^{*}\left[v_{1}, \ldots, v_{m}\right]$ is a $B P^{*}\left[x_{1}, \ldots, x_{k}\right]$-module induced by the map $\iota^{*}$. The action is given by

$$
x_{i} \cdot v=\iota^{*}\left(x_{i}\right) v
$$

Lemma 6.9. $B P^{*}\left[v_{1}, \ldots, v_{m}\right]$ is a free $B P^{*}\left[x_{1}, \ldots, x_{k}\right]$-module on $\Re_{\text {min }}$ up to dimension $\left|\Re_{\text {min }}\right|-1$.

Proof. It is clear that $B P^{*}\left[v_{1}, \ldots, v_{m}\right]$ is a free $B P^{*}\left[x_{1}, \ldots, x_{k}\right]$-module in the range where a relation among relations does not occur. $\Re_{\text {min }}$ appears in degree $\left|\Re_{\text {min }}\right|$. Hence, $B P^{*}\left[v_{1}, \ldots, v_{m}\right]$ is a free $B P^{*}\left[x_{1}, \ldots, x_{k}\right]$-module in degrees less than the degree of $\Re_{\min }$.

We now have the following

Theorem 6.10.

$$
B P^{*}\left[x_{1}, \ldots, x_{k}\right] \stackrel{\iota^{*}}{\longrightarrow} B P^{*}\left[v_{1}, \ldots, v_{m}\right] \longrightarrow B P^{*}\left(B_{T} P\right)
$$

is a projective extension sequence up to dimension: $\left|\Re_{\min }\right|-1$.

Proof. Resolve the $B P$-face ring in the category of $B P_{*}$-algebras to give the following diagram

$$
B P^{*}\left[x_{1}, \ldots, x_{k}\right] \stackrel{\iota^{*}}{\longrightarrow} B P^{*}\left[v_{1}, \ldots, v_{m}\right] \longrightarrow B P^{*}\left(B_{T} P\right) .
$$

The result follows from Definitions 5.2, 5.4, Remark 5.5 and Lemma 6.9.

We apply the functor $\operatorname{Hom}\left(-, B P_{*}\right)$ to the projective extension sequence to obtain the following injective extension sequence:

$$
B P^{*}\left(B_{T} P\right)^{\sharp} \longrightarrow B P^{*}\left[v_{1}, \ldots, v_{m}\right]^{\sharp} \stackrel{\iota^{*}}{\longrightarrow} B P^{*}\left[x_{1}, \ldots, x_{k}\right]^{\sharp}
$$

up to dimension $\left|\Re_{\min }\right|-1$. For convenience we make the following changes in notation:

$$
\begin{aligned}
C^{*} & =B P^{*}\left(\prod_{m} C P^{\infty}\right), \\
F^{*} & =B P^{*}\left(B_{T} P\right), \\
R^{*} & =B P^{*}\left[x_{1}, \ldots, x_{k}\right] .
\end{aligned}
$$

We write bases for these algebras over $B P_{*}$. We have the monomial basis for $C^{*}$ :

$$
B_{C^{*}}=\left\{v^{I} \mid I=\left(i_{1}, \ldots, i_{m}\right)\right\},
$$

a basis for $F^{*}$

$$
B_{F^{*}}=\left\{v^{I} \mid \operatorname{im} \iota^{*} \nmid v^{I}\right\},
$$


and the monomial basis for $R^{*}$

$$
B_{R^{*}}=\left\{x^{I} \mid I=\left(i_{1}, \ldots, i_{k}\right)\right\} .
$$

We dualize to co-algebras so that we can compute the higher-derived functors of the primitive element functor of the Borel space using the techniques described in [Bous]. For notational convenience we will not use lower case $*$ to denote the dual co-algebras. We write $C, F$ and $R$. To determine the bases of these co-algebras we consider the canonical pairings

$$
B P^{*}(-) \otimes \operatorname{Hom}\left(B P^{*}(-), B P_{*}\right) \rightarrow B P_{*}
$$

For example,

$$
B P^{*}\left[v_{1}, \ldots, v_{m}\right] \otimes \operatorname{Hom}\left(B P^{*}\left[v_{1}, \ldots, v_{m}\right], B P_{*}\right) \rightarrow B P_{*}
$$

is the canonical pairing for $C^{*}$. Define $\left\{\beta_{J}\right\}$ to be the basis dual to $B_{C^{*}}$ given by $\left\langle v^{I}, \beta_{J}\right\rangle=\delta_{I, J}$, and $\left\{\lambda_{J}\right\}$ the basis dual to $B_{F^{*}}$ given by $\left\langle v^{I}, \lambda_{J}\right\rangle=\delta_{I, J}$, where $I$ and $J$ are subject to the condition defining the basis for $F^{*}$. Finally, let $\left\{z_{J}\right\}$ be the basis dual to $B_{R^{*}}$ given by $\left\langle x^{I}, z_{J}\right\rangle=\delta_{I, J}$.

Example 6.11. To illustrate how the algebras and co-algebras are dual we suppose $K$ is the simplicial complex:

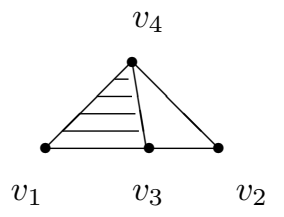

The $B P^{*}$-face ring is $B P_{*}\left[v_{1}, v_{2}, v_{3}, v_{4}\right] / I=\left\langle v_{1} v_{2}, v_{2} v_{3} v_{4}\right\rangle$. Clearly, $C^{*} \cong$ $B P^{*}\left\{v^{I} \mid \iota_{j} \geqslant 0\right\}$ and $F^{*} \cong C^{*} / I$. $R^{*}$ is a polynomial algebra whose generators are in a one to one correspondence with the relations. $R^{*} \cong B P^{*}\left\{x_{1}^{\iota_{1}} x_{2}^{\iota_{2}} \mid \iota_{1}, \iota_{2} \geqslant 0\right\}$. The Kronecker pairing $\langle$,$\rangle is used to write down the dual basis elements for the co-$ algebras. For example, $C \cong B P_{-*}\left\{\beta_{J} \mid J=\left(\iota_{1}, \ldots, \iota_{4}\right)\right\}$. For the co-algebra $F$, we have, for example, the element $\lambda_{(1,0,1,0)}$ which is dual to $v_{1} v_{3} \in F^{*}$. The bases in $F$ and $R$ can be written down in a similar way as well as any dual elements.

Let $l(J)=\sum_{i=1}^{l} j_{i}$ for $j_{i} \in J$. The following example will illustrate how one computes the map $F \rightarrow C$ which comes from resolving the ideal $I$ or more explicitly, from $B_{T} P \rightarrow \prod_{m} \mathbb{C} P^{\infty}$.

Example 6.12. Suppose $M=\{1, \ldots, q\}$ is a missing face of $K$. The element $v_{1} \cdots v_{q}$ $=0$ in $F^{*}$. In particular $v_{1} \cdots v_{q}$ is not a basis element in $F^{*}$ which implies that $\iota^{*}(x)=v_{1} \cdots v_{q}$ for a basis element $x \in R^{*}$. It follows that

$$
\lambda_{\underbrace{(1, \ldots, 1}_{q-\text { terms }}, 0, \ldots, 0)}
$$

does not exist in the co-algebra $F$. 
The degrees of the elements in the dual bases are as follows:

$$
\begin{gathered}
\left|\beta_{J}\right|=2 \cdot l(J), \\
\left|\lambda_{J}\right|=\left|v^{I}\right| \quad \text { where }\left\langle v^{I}, \lambda_{J}\right\rangle=\delta_{I J} \text { and im } \iota^{*} \nmid v^{I},
\end{gathered}
$$

and

$$
\left|z_{J}\right|=\left|\iota^{*}\left(x^{I}\right)\right| .
$$

Example 6.13. In Example 6.11 the element $z_{(1,0)} \in R$ is dual to $x_{1} \in R^{*}$ and $z_{(0,1)} \in$ $R$ is dual to $x_{2} \in R^{*}$ by the Kronecker pairing. Therefore,

$$
\left|z_{(1,0)}\right|=\left|x_{1}\right|=\left|\iota^{*}\left(x_{1}\right)\right|=\left|v_{1} v_{2}\right|=4
$$

and, similarly, we have

$$
\left|z_{(0,1)}\right|=\left|x_{2}\right|=\left|\iota^{*}\left(x_{2}\right)\right|=\left|v_{2} v_{3} v_{4}\right|=6 .
$$

The co-algebra structure maps are defined as follows;

$$
\begin{gathered}
C \stackrel{\psi}{\longrightarrow} C \otimes_{B P_{*}} C, \\
\psi\left(\beta_{I}\right)=\sum_{\substack{j_{p}+k_{p}=i_{p} \\
1 \leqslant p \leqslant m}} \beta_{j_{1}, \ldots, j_{m}} \otimes \beta_{k_{1}, \ldots, k_{m}} .
\end{gathered}
$$

The co-algebra map $R$ is defined similarly. The co-algebra map for $F$ can be defined with restrictions on the multi-indices as illustrated by the following.

Remark 6.14. Given $F$ we have

$$
\lambda_{I} \rightarrow \sum a_{K J} \lambda_{K} \otimes \lambda_{J} .
$$

The coefficients are given by

$$
\begin{aligned}
a_{K J} & =\left\langle\psi\left(\lambda_{I}\right), v^{K} \otimes v^{J}\right\rangle \\
& =\left\langle\lambda_{I}, v^{K+J}\right\rangle .
\end{aligned}
$$

Hence,

$$
a_{K J}= \begin{cases}1, & \text { if } I=K+J \\ 0, & \text { otherwise. }\end{cases}
$$

Let $A$ be an algebra. The module of indecomposables of $A, Q A=A / A A$, where $A A=\{x \in A \mid x=y z: y, z \in A\}$.

Theorem 6.15. Given a simple polyhedral complex $P$, then up to dimension $\left|\Re_{\text {min }}\right|-1$, the $B P_{*}$-module structure of $R^{i} P B P_{*}\left(B_{T} P\right)$ is as follows:

$$
R^{i} P B P_{*}\left(B_{T} P\right)^{\sharp} \cong \begin{cases}B P_{*}\left\{a_{1}, \ldots, a_{m}\right\}, & i=0,\left|a_{i}\right|=2 \\ B P_{*}\left\{e_{1}, \ldots, e_{k}\right\}, & \text { if } i=1,\left|e_{j}\right|=\left|r_{j}\right| \\ 0, & \text { otherwise. }\end{cases}
$$


Proof. By Theorem 6.10,

$$
R^{*} \stackrel{\iota^{*}}{\longrightarrow} C^{*} \longrightarrow F^{*}
$$

is a projective extension through $\left|\Re_{\min }\right|-1$. Dually,

$$
F \longrightarrow C \stackrel{\iota^{a} s t}{\longrightarrow} R
$$

is an injective extension sequence through the range $\left|\Re_{\text {min }}\right|-1$ giving rise to the following long exact sequence

$$
0 \longrightarrow P F \longrightarrow P C \longrightarrow P R \longrightarrow R^{1} P F \longrightarrow R^{1} P C \longrightarrow \cdots
$$

Since $C$ is cofree as a co-algebra we obtain the exact sequence

$$
0 \longrightarrow P F \longrightarrow P C \longrightarrow P R \longrightarrow R^{1} P F \longrightarrow 0
$$

through the range $\left|\Re_{\min }\right|-1$. By the neighborliness of $P$, the generators of $R^{*}$ are at least degree four. The generators of $C^{*}$ are of degree two. Hence, $Q R^{*} \rightarrow Q C^{*}$ factors through zero which implies that the map $P C \rightarrow P R$ factors through zero. Since $R^{1} P F$ is the co-kernel of the map $P C \rightarrow P R$ the result follows.

Remark 6.16. We will show that there is a Künneth theorem in the category of unstable $G$-co-algebras if we restrict attention to the bottom-dimensional classes. More specifically, if $M$ and $N$ are free $B P_{*}$-modules on even-dimensional generators, then the element $\bar{x} \otimes \bar{y} \in G(M) \otimes G(N)$ corresponds to the element $\bar{x} * \bar{y} \in G(M \otimes N)$. This is an external star product which comes from the completed tensor product $\widehat{\otimes}$.

Let $X$ and $Y$ be topological spaces with free $B P_{*}$-homology. Let $\mathrm{M}$ and $\mathrm{N}$ be the $B P$-homology of $X$ and $Y$, respectively, where $M=B P_{*}\left\{x_{1}, \ldots, x_{2 k}\right\}$ and $N=$ $B P_{*}\left\{y_{1}, \ldots, y_{2 k}\right\}$ such that the generators are even-dimensional. For each generator of $M$ and $N$ there is a copy of $\underline{B P}_{\left|x_{2 k}\right|}$ and $\underline{B P}_{\left|y_{2 j}\right|}$ in $G(M)$ and $G(N)$. Recall that the lowest-dimensional generator $b_{(0)}^{i} \in B P_{2 i}\left(\underline{B P}_{2 i}\right)$. Let $\bar{x}=b_{(0)}^{|x| / 2} \in B P_{*}\left(\underline{B P}_{|x|}\right)$, which is the bottom-dimensional generator for the copy of $B P_{*}\left(\underline{B P}_{|x|}\right)$ corresponding to $x$ in $G(M)$. For each module we can define a linear map on the generators $\rho: M \rightarrow G(M)$ by $\rho(x)=\bar{x}$. Similarly, there is a class $b_{(0)}^{|y| / 2} \in B P_{*}\left(\underline{B P}_{|y|}\right)$ for each generator of $N$ and a map can be defined from $N \rightarrow G(N)$. To state the next theorem we need to set up some notation. Given modules $M$ and $N$ as above we have $G(M)=B P_{*}\left(\prod_{x_{i} \in M} \underline{B P}_{\left|x_{i}\right|}\right)$ and similarly $G(N)=B P_{*}\left(\prod_{y_{i} \in N} \underline{B P}_{\left|y_{i}\right|}\right)$. We have $G(M) \otimes G(N)=B P_{*}\left(\prod_{x_{i} \in M} \underline{B P}_{\left|x_{i}\right|}\right) \otimes B P_{*}\left(\prod_{y_{i} \in N} \underline{B P}_{\left|y_{i}\right|}\right)$, which is equal to $B P_{*}\left(\prod \underline{B P}_{\left|x_{i}\right|} \times \underline{B P}_{\left|y_{i}\right|}\right)$. Note that $G(M \otimes N)=G\left(B P_{*}(X \times Y)\right)$.

Theorem 6.17. On the generators of $M$ and $N$ there exists a map

$$
\kappa_{\mathcal{G}}: G(M) \otimes G(N) \rightarrow G(M \otimes N)
$$

that sends $\bar{x} \otimes \bar{y}$ to the completed tensor product $\bar{x} \widehat{\otimes} \bar{y}$.

Proof. Given $M$ and $N$ as above let $\{x\}$ and $\{y\}$ be families of generators. We can define a map

$$
B P_{*}(X) \otimes B P_{*}(Y) \stackrel{\rho \otimes \rho}{\longrightarrow} G(M) \otimes G(N)
$$

by $(\rho \otimes \rho)(x \otimes y)=\bar{x} \otimes \bar{y}$ on the generators. By the Künneth theorem we have the 
isomorphism

$$
B P_{*}(X) \otimes B P_{*}(Y) \stackrel{\kappa}{\longrightarrow} B P_{*}(X \times Y) .
$$

Define a map

$$
B P_{*}(X \times Y) \stackrel{\bar{\rho}}{G}(M \otimes N)
$$

by

$$
\bar{\rho}(x \widehat{\otimes} y)=\rho(x) \widehat{\otimes} \rho(y)=\bar{x} \widehat{\otimes} \bar{y},
$$

where $\bar{x} \widehat{\otimes} \bar{y}$ is the bottom generator of the factor $B P_{|x \otimes y|}$. Note that $\widehat{\otimes}$ is the completed tensor product and $x \in B P_{*}(X), y \in B P_{*}(Y)$. The loop space product (Pontryagin product) will be denoted by the $*$ product. Hence, $\bar{x} \widehat{\otimes} \bar{y}=\bar{x} * \bar{y}$. Define $\kappa_{\mathcal{G}}(\bar{x} \otimes \bar{y})=$ $\bar{\rho}(x \widehat{\otimes} y)$ giving the following commutative diagram:

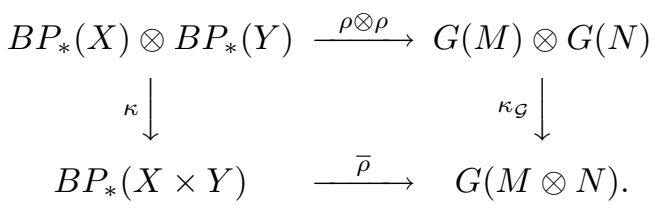

$\kappa_{\mathcal{G}}$ depends on the basis and maps the product of the generators to the generator of the factor of the product corresponding to the tensor product of the generators.

By $x \in B P_{*}(X \times Y)$ we mean $x \widehat{\otimes} 1$ and similarly $y$ means $1 \widehat{\otimes} y$. In $G(M) \otimes G(N)$, $\bar{x}$ corresponds to $\bar{x} \otimes 1$. In $G(M \otimes N), \bar{x}$ corresponds to $\bar{x} * 1$; hence

$$
\begin{aligned}
\bar{x} * \bar{y} & =(\bar{x} \widehat{\otimes} 1) *(1 \widehat{\otimes} \bar{y}) \\
& =(\bar{x} * 1) \widehat{\otimes}(1 * \bar{y}) \\
& =\bar{x} \widehat{\otimes} \bar{y} .
\end{aligned}
$$

The class $\bar{x} \otimes \bar{y} \neq \overline{x \otimes y}$. This is the difference between the class in

$$
B P_{*}\left(\underline{B P}_{|x|} \times \underline{B P}_{|y|}\right) \text { and } B P_{*}\left(\underline{B P}_{|x \otimes y|}\right) .
$$

Remark 6.18. The previous theorem does not say anything about the higher-dimensional classes.

We summarize the above with the

Remark 6.19. $\bar{x} \otimes \bar{y} \in G(M) \otimes G(N)$ corresponds to the element $\bar{x} * \bar{y} \in G(M \otimes N)$ and this is an eternal $*$ product that arises from chasing the diagram

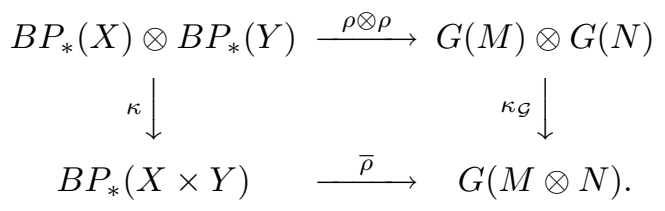

We want to apply this to $C$. Since $B P_{*}\left(\mathbb{C} P^{\infty}\right)$ is torsion free we have

$$
B P_{*}\left(\prod_{m} C P^{\infty}\right) \cong \bigotimes_{m} B P_{*}\left(C P^{\infty}\right)
$$

by the Künneth theorem. Suppose for each $j, X_{j}$ is a space such that $B P_{*}\left(X_{j}\right)$ is 
torsion free and is generated by even-dimensional generators. We have the following lemma whose proof we omit.

Lemma 6.20. The following diagram commutes.

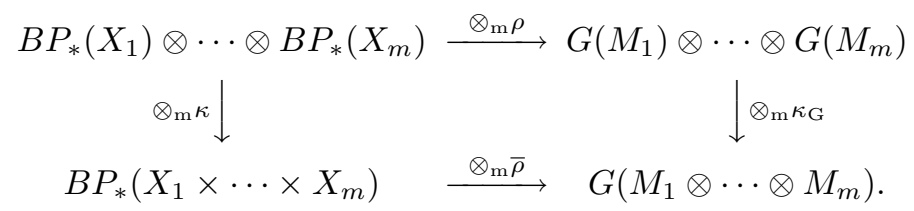

The element $\bar{x}_{1} \otimes \cdots \otimes \bar{x}_{m} \in G\left(M_{1}\right) \otimes \cdots \otimes G\left(M_{m}\right)$ corresponds to the element $\bar{x}_{1} *, \cdots, * \bar{x}_{m} \in G\left(M_{1} \otimes \cdots \otimes M_{m}\right)$. Let $I$ and $J$ be a multi-indices of non-negative integers $\left(\iota_{1}, \ldots, \iota_{m}\right)$ and $\left(j_{1}, \ldots, j_{m}\right)$. From previous arguments $\left\langle v^{I}, \beta_{J}\right\rangle=\delta_{I J}$, where $v^{I}$ are elements of $C^{*}$ and $\beta_{J}$ are elements of the dual co-algebra $C$, respectively. We can re-write the Kronecker pairing in the following way $\left\langle v^{\left(\iota_{1}, \ldots, \iota_{m}\right)}, \beta_{\left(j_{1}, \ldots, j_{m}\right)}\right\rangle$ which is the same as $\left\langle v_{1}^{\iota_{1}} \otimes \cdots \otimes v_{m}^{\iota_{m}}, \beta_{\left(j_{1}, \ldots, j_{m}\right)}\right\rangle$. Since $\langle-,-\rangle$ respects the tensor product we obtain $\left\langle v_{1}^{\iota_{1}}, \beta_{j_{1}}\right\rangle \otimes \cdots \otimes\left\langle v_{m}^{\iota_{m}}, \beta_{j_{m}}\right\rangle$. It follows that

$$
\beta_{J}=\beta_{j_{1}} \otimes \beta_{j_{2}} \otimes \cdots \otimes \beta_{j_{m}} .
$$

We introduce the following notation.

Definition 6.21. $\beta_{1, j}=\beta_{(0, \ldots, 0,1,0, \ldots, 0)}$, where 1 is in the $j^{\text {th }}$ spot.

Remark 6.22. $\beta_{1, j}$ is not to be confused with a multi-index which is enclosed in parenthesis.

$\beta_{1, j}$ generates the $j^{\text {th }}$ factor of $B P_{*}\left(C P^{\infty}\right)$ in the tensor decomposition of $C$.

Determination of the stable and unstable co-action

Lemma 6.23. $\psi: C \rightarrow \Gamma \bigotimes_{B P_{*}} C$ sends $\beta_{1, j}$ to $1 \otimes \beta_{1, j}$, and if $I$ is a multi-index of zeros and ones, then the map

$$
\psi: C \rightarrow \Gamma \bigotimes_{B P_{*}} C
$$

sends $\beta_{I} \mapsto 1 \otimes \beta_{I}$.

Proof. Since $\beta_{1, j}$ is a generator of the $j^{\text {th }}$ factor of $C$ it is a bottom-dimensional class and the result follows. The proof follows from the fact that $\psi$ is multiplicative.

The next lemma will compute the unstable co-action on the classes $\beta_{J}$ which is a product of $\beta_{1, j}$.

Lemma 6.24. For $\psi_{G(C)}: C \rightarrow G(C), \psi_{G(C)}\left(\beta_{J}\right)=*_{j \in J} \overline{\beta_{1, j}}+1 \otimes \beta_{J}$, where $1 \otimes \beta_{J}$ is an element in $G(C)$ that is mapped to $1 \otimes \beta_{J}$ by the stabilization map $\sigma_{*}$.

Proof. Let $J$ be a multi-index of zeros and ones. The following diagram shows the relation between the unstable and stable co-actions. Note that $\sigma_{*}$ is the stabilization 
map

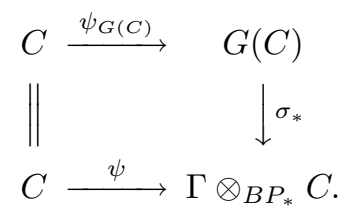

It follows from Theorem 6.17 that the image of $\psi_{G(C)}$ is given by

$$
*_{j \in J} \overline{\beta_{1, j}}+u
$$

where $u$ is a stable class. We claim that $u=1 \otimes \beta_{J}$. Since $G(C)$ and $C$ are coalgebras we have maps $\Delta_{G}: G(C) \rightarrow G(C) \otimes G(C)$ and $\Delta_{C}: C \rightarrow C \otimes C$. Using the stabilization map $\sigma_{*}$ and properties of the tensor product we have the commutative diagram:

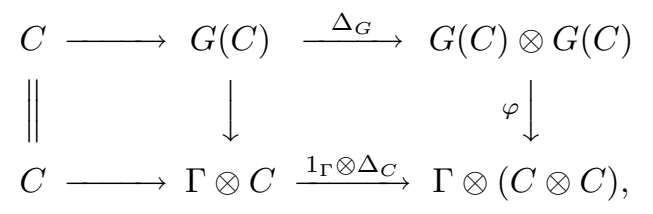

where $\varphi$ is the composition of $\sigma_{*} \otimes \sigma_{*}$ with maps that come from the associative and commutative properties of the tensor product along with the multiplication on $\Gamma$. By abuse of notation, let $\overline{\beta_{1}}$ and $\overline{\beta_{2}}$ be star products of the bottom-dimensional classes $\overline{\beta_{1, j}}$. Similarly, let $\beta_{1}$ and $\beta_{2}$ be tensor products of bottom-dimensional classes $\beta$ indexed by $J$. Now, $\Delta_{G}\left(*_{j \in J} \overline{\beta_{1, j}}+u\right)=\Sigma \overline{\beta_{1}} \otimes \overline{\beta_{1}}+\Delta_{G}(u)$. If we evaluate $\varphi$ on this class we obtain $\Delta_{G}(u)=1 \otimes\left(\sum \beta_{1} \otimes \beta_{2}\right)$ since $*$ products vanish under stabilization. By commutativity, this implies that the stable class $u$ is $1 \otimes \beta_{J}$.

Now that the higher-derived functors of the primitive element functor of the Borel space have been computed through a range, the structure of $R^{i} P B P_{*}\left(B_{T} P\right)$ as an unstable comodule in the category $A(U)$ must be determined. To do this, the generators of $R^{i} P B P_{*}\left(B_{T} P\right)$ must be represented in a way that allows for the co-action to be computed. The $G$ resolution is a useful tool that allows for one to represent these generators as external star products of bottom-dimensional classes in a particular unstable $G$-co-algebra.

$$
\begin{aligned}
0 \rightarrow \quad C \rightarrow \quad G(C) & \rightarrow \quad G^{2}(C) \\
& \rightarrow \cdots \\
& \rightarrow
\end{aligned}
$$

We apply the primitive element functor $P(-)$ to the previous chain complex to obtain the following chain complex:

$$
\begin{aligned}
(*) 0 \rightarrow U(C) & \rightarrow U(G(C)) \\
& \rightarrow \cdots \\
& \rightarrow
\end{aligned}
$$

Since $G(C)$ is cofree as a co-algebra the homology of $\left(^{*}\right)$ are the derived functors 
of the primitives i.e., $H^{i}(*)=R^{i} P(C)$. We obtain the following complexes:

$$
\begin{aligned}
\mathrm{G} & =\left\{0 \rightarrow G(C) \rightarrow G^{2}(C) \rightarrow \cdots\right\} \\
\mathrm{U} & =\{0 \rightarrow U(C) \rightarrow U(G(C)) \rightarrow \cdots\} .
\end{aligned}
$$

Since $U(C) \hookrightarrow G(C)$ we obtain the following short exact sequence of chain complexes:

$$
0 \longrightarrow \mathrm{U} \longrightarrow \mathrm{G} \longrightarrow \mathrm{G} / \mathrm{U} \longrightarrow
$$

Taking homology we obtain the following exact sequence:

$$
0 \longrightarrow H^{0} \mathrm{U} \longrightarrow H^{0} \mathrm{G} \longrightarrow H^{0} \mathrm{G} / \mathrm{U} \longrightarrow H^{1} \mathrm{U} \longrightarrow H^{1} \mathrm{G} \longrightarrow 0 .
$$

Recall that the $G$ resolution is a cobar resolution which is canonically acyclic via the cosimplicial identities. For a complex $N$ we have the isomorphism $H_{\operatorname{Hom}}\left(B P_{*}, G(N)\right) \cong N$ [BDM, p. 32]. In our case, let $N$ be the un-augmented chain complex obtained from the $G$ resolution by taking the alternating sum of the coface maps. We use the isomorphism above to deduce the acyclicity of G. We have the exact sequence:

$$
0 \longrightarrow H^{0} \mathrm{U} \longrightarrow H^{0} \mathrm{G} \longrightarrow H^{0} \mathrm{G} / \mathrm{U} \longrightarrow H^{1} \mathrm{U} \longrightarrow 0
$$

Referring to the $\mathrm{U}$ and $\mathrm{G}$ complexes of $C$ we use exactness to determine

$$
H^{0} \mathrm{G}=\text { ker }: G(C) \longrightarrow G^{2}(C)
$$

and

$$
H^{0} \mathrm{U}=\text { ker }: U(C) \longrightarrow U(G(C)) .
$$

$H^{0} \mathrm{G}=C$ and $H^{0} \mathrm{U}=P C$. As a matter of notational convenience, let

$$
\phi:\left[\frac{G(C)}{U(C)} \rightarrow \frac{G^{2}(C)}{U(G(C))}\right]
$$

The $\operatorname{ker} \phi$ is $H^{0} \mathrm{G} / \mathrm{U}$. $\mathrm{U}$ is a chain complex whose homology groups are the derived functors of the co-algebra $C$. We immediately obtain the following long exact sequence:

$$
0 \longrightarrow>P C \longrightarrow C \stackrel{\theta}{\longrightarrow} H^{0} \mathrm{G} / \mathrm{U} \stackrel{h}{\longrightarrow} R^{1} P C \longrightarrow 0 .
$$

The map $\theta$ factors in the following way

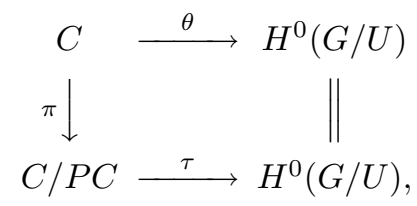

where $\tau=\theta \circ \pi^{-1}$.

Proposition 6.25. $R^{1} P(C) \cong \operatorname{ker} \phi$ modulo im $\frac{C}{P C}$.

Proof. The map $h: H^{0} \mathrm{G} / \mathrm{U} \rightarrow R^{1} P C$ is surjective by the previous long exact sequence. The first isomorphism theorem gives $\frac{\operatorname{Ker} \phi}{\operatorname{Ker} h} \cong R^{1} P(C)$. The result follows from the factorization of $\theta$. 
There is a shift that comes from the chain complex $(*)$ and the chain complexes that follow by utilizing the isomorphism $\operatorname{Hom}_{\mathcal{G}}\left(B P_{*}, G(N)\right) \cong N$. For example,

$$
\frac{G^{2}(C)}{U(G(C))}=\frac{G^{1}(C)}{U^{1}(C)}
$$

If $C$ were $B P_{*}\left(\prod_{m} \mathbb{C} P^{\infty}\right)$, then $R^{1} P C=0$ and the previous constructions would not yield any new information. However, $R^{1} P F \neq 0$. Hence, the constructions above would be applied to the co-algebra $F$. Elements in $R^{1} P F$ must be represented in a way that will allow for the determination of the unstable $U$-comodule structure map.

Recall that the data needed to work with the CFSS consists of the $R^{i} P(-)$ as well as the unstable and stable co-actions. We will show that through a range the generators of $R^{i} P F$, where $F=B P_{*}\left(B_{T} P\right)$ can be written as certain star products in $G(F)$, then determining the unstable co-action reduces to determination of the map $G(F) \rightarrow U(G(F))$.

Theorem 6.26. $d: G(F) \rightarrow U(G(F))$ is given by $d\left(*_{j \in J} \overline{\beta_{1, j}}\right)=1 \otimes\left(*_{j \in J} \overline{\beta_{1, j}}\right)$.

Proof. Consider the following commutative diagram with exact rows.

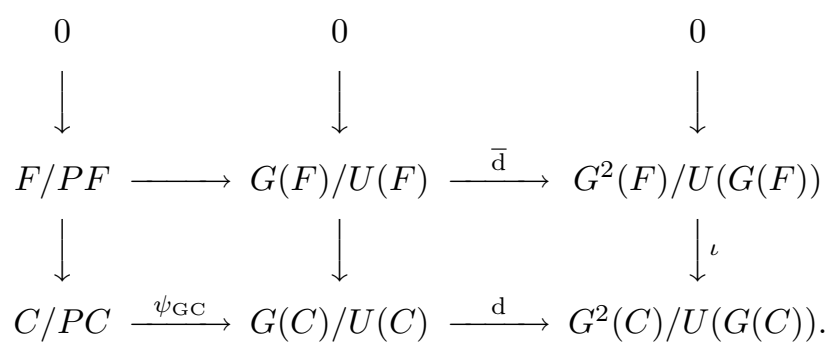

By Lemma 6.24, $\psi_{G(C)}\left(\beta_{J}\right)=*_{j \in J} \overline{\beta_{1, j}}+1 \otimes \beta_{J}$. Observe that $d \circ \psi_{G(C)}=0$. Since $d$ is a map of spaces we can write $d\left(*_{j \in J} \overline{\beta_{1, j}}\right)=-d\left(1 \otimes \beta_{J}\right)$. There exists a a class $\bar{\alpha} \in G(F) / U(F)$ which hits $*_{j \in J} \overline{\beta_{1, j}}+1 \otimes \beta_{J}$. We assert that $\bar{\alpha}=*_{j \in J} \overline{\beta_{1, j}}$. Notice the element $1 \otimes \beta_{J}$ cannot pullback since $F$ does not have a product structure map. If $\bar{\alpha}$ is a class that is not divisible by $*_{j \in J} \overline{\beta_{1, j}}$, then it pulls back to a class in $P / P F$, which would violate the commutativity of the first square. Suppose that $\bar{\alpha}$ is a class divisible by $*_{j \in J} \overline{\beta_{1, j}}$, but not the class $*_{j \in J} \overline{\beta_{1, j}}$, then we use injectivity to show that $\bar{\alpha}$ is as claimed. By commutativity we observe that $(\iota \circ \bar{d})\left(*_{j \in J} \overline{\beta_{1, j}}\right)=0$. Since $\iota$ is an injection, $\bar{d}\left(*_{j \in J} \overline{\beta_{1, j}}\right)=0$. Hence, $*_{j \in J} \overline{\beta_{1, j}}$ is a non-trivial cycle that represents an element in $R^{1} P F$ which cannot be pulled back to $F / P F$. We immediately have the following commutative diagram:

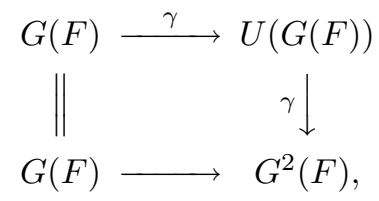

where $\gamma$ is the lift one obtains from $*_{j \in J} \overline{\beta_{1, j}}$ being a cycle. The map $d\left(1 \otimes \beta_{J}\right)$ must be determined. In filtration zero in the unstable cobar complex of $G^{s}(\Gamma \otimes C)$ we have 
$d\left(1 \otimes \beta_{J}\right)=1 \otimes 1 \otimes \beta_{J}-1 \otimes \psi_{G C}\left(\beta_{J}\right)$. Hence, by Lemma 6.24 we have

$$
d\left(1 \otimes \beta_{J}\right)=-1 \otimes\left(*_{j \in J} \overline{\beta_{1, j}}\right)
$$

giving the result.

If we define a bi-cosimplicial object $D^{p, q}(F)=U^{p} G^{q}(F)$ with $q=1, p \geqslant 0$, then it becomes clear that we can realize the map $G(F) \rightarrow U(G(F))$. If all the primitives are in the same degree then the triviality of the co-action follows from degree reasons. The content of the earlier part of this section is to deal with mixed degrees.

The map between $F$ and $C$ is induced from a map of spaces. This allows for the co-action on $F$ to be deduced from the co-action on $C$.

Corollary 6.27. The $\Gamma$-co-action on $F$ is trivial.

Proof. The map of co-algebras $F \rightarrow C$ is injective. The image of $F$ in $C$ is a coalgebra with basis consisting of those $\left\{\beta_{J}\right\}$ such that the dual basis elements $\left\{v^{I}\right\}$ are not divisible by im $\iota^{*}$. The result follows from Proposition 6.23.

Since the algebra $R^{*}$ is not realized as the $B P$-co-homology of a space, the $\Gamma$-coaction on $R$ cannot be deduced from the co-action on $C$ or $F$. The map $C \rightarrow R$ is not induced from a map of spaces.

Theorem 6.28. The $\Gamma$-co-action on $R$ is trivial.

Proof. There are two cases to analyze. First, suppose that $\forall i \neq j,\left|x_{i}\right|=\left|x_{j}\right|$ in $R$. The co-action must be trivial by dimensional reasons since $\psi(x)=1 \otimes x+\sum \gamma_{i} \otimes x_{i}$, where $\operatorname{deg}\left(\gamma_{i}\right)>0$ and $\operatorname{deg}\left(x_{i}\right)<\operatorname{deg}(x)$. Now suppose the degrees of the relations are not the same. Hence, there are multi-indexes $J$ and $J_{i}$ consisting of zeros and ones such that for each $i,\left|z_{J_{i}}\right|<\left|z_{J}\right|$, where $z_{J_{i}}$ and $z_{J}$ are primitives in $R$. Let $p$ be a prime. Suppose there is a possible co-action

$$
\psi\left(z_{J}\right)=1 \otimes z_{J}+\epsilon_{1} \cdot h^{I} \otimes z_{J_{1}}+\cdots+\{\text { Other Terms }\} .
$$

By Theorem 6.15, $P R$ is isomorphic to $R^{1} P F$ as $B P_{*}$-modules within a specified range. By the Kronecker pairing, $z_{J_{i}}$ is dual to $x^{I_{i}}$, which maps to products of $v$ 's coming from $x^{I_{i}}$ via the map $\iota^{*}$. Recall that $v^{I_{i}}$ are dual to elements $\beta_{J_{i}} \in C$ that can be written as $*$ products of bottom-dimensional classes in $G(F)$ by Theorem 6.26 . Also recall that the classes $\overline{\beta_{i, j}}$ in $G(F)$ can be identified as a $\otimes$ product and that they come from various factors of $\mathbb{C} P^{\infty}$.

The co-action on $R$ is computable since the relations come from the square-free monomials that generate the ideal in the face ring. The co-action on the $\beta_{i, j}$ is trivial by the geometry since they come from the $v_{i}$ that come from $\mathbb{C} P^{\infty}$. If the relations did not come from square-free monomials, then $\beta_{J}$ is an element of $C$ whose multiindex does not consist of zeros and ones. Without the hypothesis of square-free, Lemma 6.23 does not necessarily apply. Closer inspection of Lemmas 6.23 and 6.24 shows that square-free is reflected in the multi-indexes $I$ and $J$ consisting of zeros and ones. The next example shows how the failure of square-free is reflected in terms of the co-action. In this case, the primitives in the co-algebra of relations $R$ have multiindexes consisting of zeros and ones. However, the elements that the primitives map 
to via $\iota^{*}$ are dual to $\beta_{J}$, where $J$ is a multi-index that consists of positive integers other than ones and zeros. It is not clear how the co-action would be computed. Compare with Lemma 6.23.

Example 6.29. Consider the element $v_{1}^{2} v_{2}$ which is represented in $C$ by the element $\beta_{(2,1,0,0)}$. At the prime $p=2$ we have the possible non-trivial co-action:

$$
\psi\left(\beta_{(2,1,0,0)}\right)=1 \otimes \beta_{(2,1,0,0)}+\epsilon_{1} \cdot h^{I_{1}} \otimes \beta_{J_{1}}+\epsilon_{2} \cdot h^{I_{2}} \otimes \beta_{J_{2}}+\{\text { other terms }\},
$$

where $\epsilon_{i}=1$ or 0 . We are not able to determine whether the coefficients $\epsilon_{i}$ are zero.

If there is a possible non-trivial co-action it is possible to pick a prime $p$ that will force the co-action to be trivial for dimensional reasons. One readily sees that the co-action in the previous example is trivial if the prime is large enough. However, this is much weaker than the argument given in the previous theorem. The following example should be illuminating.

Example 6.30. Let $K$ be the simplicial complex given in Example 6.11. Recall that the $B P^{*}$-face ring is the algebra $F^{*}=B P^{*}\left[v_{1}, \ldots, v_{4}\right] / I=\left\langle v_{1} v_{2}, v_{2} v_{3} v_{4}\right\rangle$. As a $B P_{*^{-}}$ module, the co-algebra of relations $R$ has the following presentation.

$$
R=B P_{-*}\left\{z_{J} \mid J=\left(j_{1}, j_{2}\right)\right\} .
$$

For $p>2$ prime there cannot be a stable co-action for dimensional reasons. However at the prime $p=2$ there is a possible co-action:

$$
\psi\left(z_{(0,1)}\right)=1 \otimes z_{(0,1)}+\epsilon \cdot h_{1} \otimes z_{(1,0)} .
$$

The primitives in $R$ can be represented by the following products in $G(F)$ :

$$
z_{(1,0)}=\overline{\beta_{1,1}} * \overline{\beta_{1,2}}
$$

and

$$
z_{(0,1)}=\overline{\beta_{1,2}} * \overline{\beta_{1,3}} * \overline{\beta_{1,4}} .
$$

We have $\psi\left(z_{(0,1)}\right)=\psi\left(\overline{\beta_{1,2}} \otimes \overline{\beta_{1,3}} \otimes \overline{\beta_{1,4}}\right)=1 \otimes\left(\overline{\beta_{1,2}} \otimes \overline{\beta_{1,3}} \otimes \overline{\beta_{1,4}}\right)=1 \otimes z_{(0,1)}$ and so $\epsilon=0$. Therefore, the stable co-action is trivial. In lieu of the previous remark, the co-action is trivial for a prime $p>2$ for dimensional reasons.

\section{The Borel space and the UANSS}

The UANSS based on a theory other than ordinary homology theory was first constructed in $[\mathbf{B C M}]$. The $E_{2}$-term was identified as an Ext in a certain non-abelian category. The category of unstable $G$-co-algebras was constructed and a particular CFSS whose $E_{2}$ converges to the $E_{2}$-term of the UANSS was explicitly determined. The results of $[\mathbf{R W}]$ allowed for one to compute the $E_{2}$-term of the UANSS as the homology of the unstable cobar complex. In this section the $E_{2}$-term of the CFSS defined in $[\mathbf{B C M}]$ will be computed through a range. Let $X$ be a space such that $B P_{*}(X) \cong B P_{*}\left\{x_{2 k} \mid k>0\right\}$ and call it $M$. Note that the generators are evendimensional.

Proposition 6.31. For every $i \geqslant 0, R^{i} P M$ are generated by even-dimensional classes. 
Proof. Take the $G$ resolution of $M$ :

$$
0 \rightarrow M \rightarrow G(M) \rightarrow G^{2}(M) \rightarrow \cdots
$$

and the augmented chain complex to give:

$$
G(M) \rightarrow G^{2}(M) \rightarrow \cdots .
$$

Apply the primitive element functor $P(-)$ to give the complex:

$$
U(M) \rightarrow U(G(M)) \rightarrow U\left(G^{2}(M)\right) \rightarrow \cdots
$$

Recall that

$$
U(M)=B P_{*}-\operatorname{span}\left\{h^{I} \otimes x|2 \cdot l(I)<| x \mid\right\} .
$$

Clearly, the degree of $h^{I} \otimes x$ is even. Since $M \cong B P_{*}\left\{x_{2 k} \mid k>0\right\}$ as a $B P_{*}$-module, $G(M)=B P_{*}\left(\prod \underline{B P}_{\left|x_{i}\right|}\right)$ which is $\bigotimes_{i} B P_{*}\left(\underline{B P}_{\left|x_{i}\right|}\right)$. Hence, $U(G(M))$ is generated by classes of the form $h^{I} \otimes\left(\otimes_{i} x_{i}\right)$, which are clearly even-dimensional. We will prove by induction over $k$ that $U\left(G^{k}(M)\right)$ is generated by even-dimensional classes. Suppose that $U\left(G^{k-1}(M)\right)$ is generated by even-dimensional classes. Since

$$
U\left(G^{k}(M)\right)=U\left(G^{k-1}(G(M))\right)
$$

and $G(M)$ is generated by even-dimensional classes, the argument above implies that $U\left(G^{k}(M)\right)$ is generated by even-dimensional class.

This immediately gives

Corollary 6.32. If $K$ is a simplicial complex and $F$ the $B P_{*}$-face ring of $B_{T} P$, then $R^{i} P F$ are generated by even-dimensional classes for every $i \geqslant 0$.

Recall that $F=B P_{*}\left(B_{T} P\right)$ is nice in the range $\left|\Re_{\text {min }}\right|-1$. The primitives in the co-algebra of relations $R$ are elements $z_{J_{i}}$, where $J_{i}$ is a multi-index consisting of one in the $i^{\text {th }}$ spot and zero elsewhere. Recall that $\left|z_{J_{i}}\right|$ is equal to the degree of the products of $v$ 's that the dual element $x^{I_{i}}$ map to via $\iota^{*}$. We have the following

Theorem 6.33. If $K$ is a simplicial complex and $F$ is the $B P_{*}$-face ring associated to $P$, then in filtration $s \geqslant 0$ and total degree $t \leqslant s(2 p-2)+\left|\Re_{\text {min }}\right|-1$. For $i=0,1$, the classes in

$$
\operatorname{Ext}_{\mathcal{G}}^{i+s, t}(F)
$$

are those that come from the $E_{2}$-terms:

$$
\operatorname{Ext}_{A(U)}^{s, t}\left(B P_{*}\left(\prod_{r_{j} \in I} S^{\left|r_{j}\right|}\right)\right)
$$

for $i=1$ and

$$
\operatorname{Ext}_{A(U)}^{s, t}\left(B P_{*}\left\{a_{1}, \ldots, a_{k}\right\}\right)
$$

for $i=0$.

Proof. Since $B_{T} P$ is a space there exists a CFSS. By Corollary 6.32 the CFSS collapses for dimensional reasons and the classes in $E_{\infty}^{s, t}$ survive to the $E_{2}$-term of the 
UANSS. We assert that for $i=1$,

$$
\operatorname{Ext}_{A(U)}^{s, t}\left(R^{1} P B P_{*}\left(B_{T} P\right)\right) \cong \operatorname{Ext}_{A(U)}^{s, t}\left(B P_{*}\left(\prod_{r_{j} \in I} S^{\left|r_{j}\right|}\right)\right)
$$

and for $i=0$,

$$
\operatorname{Ext}_{A(U)}^{s, t}\left(P B P_{*}\left(B_{T} P\right)\right) \cong \operatorname{Ext}_{A(U)}^{s, t}\left(B P_{*}\left\{a_{1}, \ldots, a_{k}\right\}\right) .
$$

In the case $i=0$, we have $P F \cong P C$ by Theorem 6.15 and the co-action is trivial for dimensional reasons. By Theorem 6.15 the co-algebra $F$ is nice in degrees less than $\left|\Re_{\text {min }}\right|$. We assert that there is an isomorphism, in this range, of $B P_{*}-$ modules between $R^{1} P(F)$ and $B P_{*}\left(\prod_{r_{j} \in I} S^{\left|r_{j}\right|}\right)$, where the dimensions of the spheres equals the degrees of the primitives in the co-algebra $R$. By Theorem $6.15, R^{1} P F \cong$ $B P_{*}\left\{e_{1}, \ldots, e_{k}\right\}$. The degrees of the $e_{i}$ are equal to the degrees of the square-free monomials that generate the ideal $I$. Suppose there are $k$ such monomials. Let $S^{e_{i}}$ denote a $\left|e_{i}\right|$-dimensional sphere. The $B P_{*}\left(S^{e_{1}} \times \cdots \times S^{e_{k}}\right)$ is isomorphic to the module $B P_{*}\left\{\iota_{e_{1}}, \ldots, \iota_{e_{k}}\right\}$, where $\left|\iota_{e_{1}}\right|=\left|e_{j}\right|$ in the range $* \leqslant\left|\Re_{\text {min }}\right|-2$. In the case that the ideal $I$ is generated by monomials $r_{j}$ such that for all $i \neq j\left|r_{j}\right|=\left|r_{i}\right|$, then the isomorphism above, with the range restriction, shows that there is no mixing of the generators. In the case that there exists a monomial $r_{j}$ such that for all $i \neq j,\left|r_{j}\right|>\left|r_{i}\right|$, then the isomorphism above holds as long as $\left|r_{j}\right| \leqslant\left|\Re_{\min }\right|-2$. Theorem 6.26 shows that the $U$-comodule structure on $R^{1} P F$ coincides with the co-action on a sphere. This gives the following isomorphism of $E_{2}$-terms of the CFSS $\operatorname{Ext}_{A(U)}^{s}\left(R^{1} P(F)\right) \cong \operatorname{Ext}_{A(U)}^{s}\left(\left(B P_{*}\left(\prod_{r_{j} \in I} S^{\left|r_{j}\right|}\right)\right)\right.$. To obtain a least upper bound on the total degree $t$ let $s \geqslant 0$. The element $\overbrace{h_{1} \otimes \cdots \otimes h_{1}}^{s} \otimes r$ has degree $s(2 p-2)+|r|$. However, $\left|\Re_{\text {min }}\right|$ is a strict upper bound on $|r|$. This is seen by observing that $\left|\Re_{\text {min }}\right|$ is given by $2 l(m)$ where $m$ is the monomial that comes from one of the terms in the difference Definition 6.2 and $l(m)$ is defined in Definition 6.6. The condition $|r| \leqslant\left|\Re_{\text {min }}\right|-2$ ensures $l(r)<l(m)$, giving $\left|h_{1}^{\otimes_{s}} \otimes r\right| \leqslant s(2 p-2)+\left|\Re_{\text {min }}\right|-1$. The upper bound on the total degree $t$ is obvious when $s=0$.

Theorem 6.33 shows that within a specified range, one understands what classes survive to the $E_{2}$-term of the UANSS. By re-labeling the axes from $(t-s, s)$ to $(t, s)$ and using the condition on the total degree $t \leqslant s(2 p-2)+\left|\Re_{\text {min }}\right|-1$ a picture of the $E_{2}$-term of the UANSS can be obtained. There is a line of slope

$$
\frac{-\left|\Re_{\min }\right|+1}{2 p-2\left|\Re_{\min }\right|+1}
$$

through

$$
\frac{-\left|\Re_{\min }\right|+1}{2 p-2} \text { and }\left|\Re_{\min }\right|-1 .
$$

Since the UANSS is a first quadrant spectral sequence this implies that all the classes above the "line" are understood, before further analysis of the differentials. Assuming that $I=\left\langle r_{1}, \ldots, r_{k}\right\rangle$ the $E_{2}$ page of the UANSS will consist of a collection of $\eta$ towers (one for each monomial $r_{i} \in I$ ). Each of these towers will have a homotopy tower corresponding to the elements $\alpha, \eta \alpha, \eta^{2} \alpha \cdots$ with a $d_{3}$-differential such 
that $d_{3}(\alpha)=\eta^{4}$, where $\eta^{4}$ represents the element $h_{1} \otimes h_{1} \otimes h_{1} \otimes h_{1} \otimes \iota_{k}$. Hence, the elements $\eta^{4}, \eta^{5} \ldots$ in each tower will be killed by the $d_{3}$-differential (also a derivation) out of the corresponding homotopy tower. It should be noted that there is an abuse of notation in Theorem 6.33. The element $\alpha$ is called $\widetilde{\alpha}_{4 k+2}$ in [Bend2] and the $d_{3^{-}}$ differential comes out of $\widetilde{\alpha}_{4 k+2}$ into the element $\left(\eta^{3} \widetilde{\alpha}_{4 k+1}\right)$ where $\alpha_{1}=\eta$ when $k=0$. For the definition of $\widetilde{\alpha_{k}}$ and the determination of the unstable 1-line for $S^{2 n+1}$ the reader should refer to [Bend2]. Classes in stem degree $\left|\Re_{\text {min }}\right|-2$ have the possibility of surviving to $\pi_{t-s}$ localized at $p$.

Given that the classes in the UANSS are understood through a range one cannot immediately conclude homotopy computations. In fact, a careful analysis of the differentials is required. It may be possible for classes in the $E_{2}$-term to be killed by a differential. Recall that only the classes that survive to the $E_{\infty}$-term of the UANSS are the classes that contribute to the homotopy groups of $B_{T} P$. It turns out that the underlying combinatorics can be used to resolve the differentials. Given a $B P$ face ring the notion of killing relations must be developed. In what follows it will be convenient to work with the simplicial complex $K$ dual to $P$.

We need to recall certain operations on two simplicial complexes. Suppose $K_{1}$ and $K_{2}$ are simplicial complexes on $\left[m_{1}\right]$ and $\left[m_{2}\right]$ and suppose $\sigma_{i} \in K_{i}$ for $i=1,2$. Let $K_{1}$ and $K_{2}$ be as above with the property that a vertex set $S$ satisfies $S=$ $\left[m_{1}\right] \cup\left[m_{2}\right]$, where $\left[m_{1}\right] \bigcap\left[m_{2}\right]$ is not necessarily empty. The join of $K_{1}$ and $K_{2}$, $K_{1} * K_{2}=\left\{\sigma \subset S \mid \sigma=\sigma_{K_{1}} \cup \sigma_{K_{2}}, \sigma_{K_{1}} \in K_{1}, \sigma_{K_{2}} \in K_{2}\right\}$. It is important to note that $\phi \in K_{1}$. When $K_{2}$ is a point we obtain the cone on $K_{1}$, cone $\left(K_{1}\right)$. It is also the case that $\sigma * \emptyset=\sigma$. we say $K$ is $q$-neighborly if any subset of the vertex set of cardinality $q-1$ is a simplex.

Construction 6.34. Let $K$ be a simplicial complex and suppose its $B P$-face ring is

$$
B P_{*}\left[v_{1}, \ldots, v_{m}\right] / I=\left\langle r_{1}, \ldots, r_{k}\right\rangle
$$

We kill a relation by the following procedure. Pick $r_{1}=v_{\iota_{1}} \cdots v_{\iota_{q+1}}$. Split it in such a way as to produce two subcomplexes of $K$. Let $K_{1}$ be the simplicial complex on the vertex set $\left\{v_{\iota_{1}} \cdots v_{\iota_{q}}\right\}$ and let $K_{2}$ be the subcomplex on the vertex $\left\{v_{\iota_{q+1}}\right\}$. Let $\bar{K}=K_{1} * K_{2}$. It is clear that this simplicial complex will contain the simplex $\sigma=\left\{v_{\iota_{1}} \cdots v_{\iota_{q+1}}\right\}$. Form a new simplicial complex $K^{\prime}$ from $K$ and the subcomplexes, $K_{1}$ and $K_{2}$ in the following way. Let $K^{\prime}=K \cup \sigma$, where $\sigma \in \bar{K}$. It follows immediately that the $B P$-face ring of $K^{\prime}$ is

$$
B P_{*}\left[v_{1}, \ldots, v_{m}\right] / I=\left\langle r_{2}, \ldots, r_{k}\right\rangle
$$

In other words, the relation $r_{1}$ has been killed.

This construction gives a prescription for "killing" relations in the ideal $I$. In other words, one can "fill" in missing faces of a simplicial complex. This makes it possible to find simplicial maps from a certain $K$ on a vertex set, to another simplicial complex on the same vertex set with fewer missing faces. To resolve the differentials in the UANSS it is necessary to find such simplicial maps. 
Theorem 6.35. Given $P$ and total degree $t$ as in Theorem 6.33, let $B_{T} P$ be the associated Borel space. We have

$$
\pi_{t-s-1}\left(B_{T} P\right)_{(p)}=\pi_{t-s-1}\left(\left(\bigvee_{r_{j} \in I} S^{\left|r_{j}\right|-1}\right)\right)_{(p)} \text { for } t-s \leqslant\left|\Re_{\text {min }}\right|-1,
$$

and, in filtration $s=0, \pi_{2}\left(B_{T} P\right)=\mathbb{Z}^{\oplus m}$.

Proof. In filtration $s \geqslant 0$ the $E_{2}$-term of the UANSS is given by Theorem 6.33. To complete the proof it must be shown that there are no possible differentials in the UANSS. Suppose $I=\left\langle r_{1}, \ldots, r_{k}\right\rangle$ and $\left|r_{i}\right|>\left|r_{j}\right|$. Assume there is a possible nontrivial differential from the $\eta$ tower generated by a class that comes from $r_{i}$ into the tower generated by the class that comes from $r_{j}$. By Construction 6.34 it is possible to kill relations by filling in the appropriate missing face. Hence, one can find a simplicial map from $\phi: K_{m_{1}, \ldots, m_{k}} \rightarrow K_{m_{1}, \widehat{m_{i}}, \ldots, m_{k}}$ where $K_{m_{1}, \ldots, m_{k}}$ is a simplicial complex with missing faces $m_{1}, \ldots, m_{k}$ and $K_{m_{1}, \widehat{m_{i}}, \ldots, m_{k}}$ is a simplicial complex with the missing face $m_{i}$ filled in. Clearly, $\phi$ induces a map of Borel spaces which induces a map of $E_{2^{-}}$ terms of the UANSS that must commute with differentials. This implies that there is a non-trivial differential from a zero class to a non-zero class which is a contradiction. Therefore, the differential must be trivial. Hence, there are no non-trivial differentials between $\eta$ towers in the $E_{2}$-term of the UANSS. By Theorem 6.33 , the only classes that appear in stem degree are those that come from spheres via the CFSS and in the specified range there is either only one class in each stem degree or $|I|$ classes. In either case, the extension problem is trivial.

In the case that $K$ is a simplicial sphere we have the following:

Proposition 6.36. Suppose $M^{2 n}(\lambda)$ is quasi-toric manifold with orbit space P. For $4 \leqslant * \leqslant\left|\Re_{\text {min }}\right|-1$, we have

$$
\pi_{*-1}\left(M^{2 n}(\lambda)\right)_{(p)} \cong \pi_{*-1}\left(\left(\bigvee_{r_{j} \in I} S^{\left|r_{j}\right|-1}\right)\right)_{(p)} .
$$

Proof. There is a fibration $M^{2 n}(\lambda) \rightarrow B_{T} P \rightarrow B T^{n}$. Apply $\pi_{*}(-)$ and the result follows from Theorem 6.35.

As an application, a non-trivial higher homotopy group computation can be obtained.

Proposition 6.37. If $P^{2}$ is a polygon with $m \geqslant 5$ vertices and $M^{4}(\lambda)$ the corresponding family of quasi-toric manifolds, then

$$
\pi_{4}\left(\sharp_{m-2} \mathbb{C} P^{2}\right)_{(p)} \cong \pi_{4}\left(\bigvee_{r_{j} \in I} S^{3}\right)_{(p)} .
$$

Proof. For $m>4$, Orlik and Raymond $[\mathbf{O R}]$ proved that four-dimensional quasi-toric manifolds are connected sums of the Hirzebruch surface with two-dimensional complex projective space. Observe $\left|\Re_{\min }\right|=6$. The result follows from Propositions 6.36 and 6.56 . 
The case $m=4$ is handled by the toric geometry. It is well known in this case that for $*>2, \pi_{*}\left(M^{4}(\lambda)\right) \cong \pi_{*}\left(S^{3} \times S^{3}\right)$. We recall that quasi-toric manifolds do not necessarily exist over $P$ when $K$ is not a simplicial sphere. Moreover, for such $K, Z_{K}$ exists but it is not necessarily a manifold. However, the Borel space always exists.

Corollary 6.38. Let $K$ be a simplicial complex and $Z_{K}$ the associated moment angle complex. We have for, $4 \leqslant * \leqslant\left|\Re_{\min }\right|-1$,

$$
\pi_{*-1}\left(Z_{K}\right)_{(p)} \cong \pi_{*-1}\left(\left(\bigvee_{r_{j} \in I} S^{\left|r_{j}\right|-1}\right)\right)_{(p)}
$$

Proof. Follows from the fibration $Z_{K} \rightarrow B_{T} Z_{K} \rightarrow B T^{m}$ in combination with Theorem 6.35.

Recall that $P R$ are the primitives in the co-algebra $R$ that represent the relations. Using the bi-graded minimal resolution of $k(K)([\mathbf{B P 1}]$ and $[\mathbf{B P} 4])$, it is easily seen that the bottom-dimensional cells of $Z_{K}$ are in degrees $|P R|-1$. By the connectivity of $Z_{K}$ and the above we immediately obtain the

Corollary 6.39. $\pi_{*-1}\left(Z_{K}\right)_{(p)} \cong \pi_{*-1}\left(\bigvee_{r_{j} \in I} S^{\left|r_{j}\right|-1}\right)_{(p)}$

Theorem 2.15 in [BP4] states that the first non-trivial homotopy group is a free abelian group on a set whose cardinality is given by $|I|$. Notice that Corollary, 6.39 exhibits the isomorphism explicitly.

We define a simplicial complex such that the homotopy type of the associated moment angle complex can be determined by using filtration arguments in the UANSS.

Definition 6.40. For each integer $m \geqslant 4$, define

$$
r=[(m+1) / 2]
$$

where $[n]$ is the greatest integer value of $n$.

We make

Definition 6.41. Let $K_{[m]}$ be the simplicial complex on $[m]$ with the missing faces $\{1, \ldots, r\}$ and $\{r, r+1, \ldots, m\}$.

In the next section we prove some basic facts about $K_{[m]}$.

Proposition 6.42. Given the complex $K_{[m]}$, let $Z_{K_{[m]}}$ be the associated moment angle complex. The top-dimensional cell $e^{2 m-2}$ of $Z_{K_{[m]}}$ gives rise to a $\mathbb{Z}$ in $\pi_{2 m-2}$ detected by the class in the Ext-term

$$
\operatorname{Ext}_{\mathcal{A}(\mathcal{U})}^{0,\left|\Re_{\min }\right|}\left(R^{2} P F\right) .
$$


Proof. There are three Ext-terms that have the possibility of contributing classes to the $E_{2}$-term of the UANSS. First,

$$
\operatorname{Ext}_{A(U)}^{0,\left|\Re_{\min }\right|-1}\left(R^{1} P(F)\right) .
$$

This term is zero by Proposition 6.31 and Corollary 6.32. Next, consider the $E_{2}$-term,

$$
\operatorname{Ext}_{A(U)}^{1,\left|\Re_{\min }\right|}\left(R^{1} P(F)\right) \text {. }
$$

The bottom-dimensional cells of $Z_{K_{[m]}}$ are given in Theorem 6.49. The classes in $\operatorname{Ext}_{A(U)}^{1,\left|\Re_{\min }\right|}\left(R^{1} P(F)\right)$ contribute to the homotopy of

$$
S^{2 r-1} \bigvee S^{2 r-1} \quad \text { or } \quad S^{2 r-1} \bigvee S^{2 m-2 r+1}
$$

localized at $p$. No new classes that detect the cell $e^{2 m-2}$ are produced. The only way the cell $e^{2 m-2}$ is detected is by the term

$$
\operatorname{Ext}_{A(U)}^{0,\left|\Re_{\min }\right|}\left(R^{2} P(F)\right) .
$$

Notice that $\left|\Re_{\text {min }}\right|$ is outside the range where the spectral sequence is known. We have absolutely no control over $R^{2} P F$. Clearly, $\pi_{2 m-2}(F) \neq 0$. Hence, the cell $e^{2 m-2}$ must be detected. This can only happen if

$$
\operatorname{Ext}_{A(U)}^{0,\left|\Re_{\min }\right|}\left(R^{2} P(F)\right) \neq 0 .
$$

Therefore, $R^{2} P F \neq 0$.

There is no systematic way to construct an unstable $\mathcal{G}$ resolution in such a way as to get a handle on $R^{2} P$. However, the previous proposition gives the following:

Corollary 6.43. If $F$ is the BP $P_{*}$-face ring of the simplicial complex Definition 6.41, then

$$
R^{2} P B P_{*} F \cong B P_{*}\{\iota\}
$$

where $|\iota|=\left|\Re_{\text {min }}\right|$.

By Proposition 6.42 we obtain the

Corollary 6.44. Given the moment angle complex $Z_{K_{[m]}}$ of Definition 6.41 we have, for $* \leqslant\left|\Re_{\min }\right|-1$,

$$
\pi_{*-1}\left(Z_{K_{[m]}}\right)_{(p)}= \begin{cases}\pi_{*-1}\left(S^{2 r-1} \bigvee S^{2 r-1}\right)_{(p)}, & m \text { odd } \\ \pi_{*-1}\left(S^{2 r-1} \bigvee S^{2 m-2 r+1}\right)_{(p)}, & m \text { even }\end{cases}
$$

Remark 6.45. Through a range, the homotopy of the complex $Z_{K_{[m]}}$ localized at a prime looks like a wedge of spheres by the classes contributed by $\operatorname{Ext}_{A(U)}^{s, t}\left(R^{1} P(F)\right)$.

The homotopy type of the moment angle complex associated to certain simplicial complexes

In this section we prove some facts about the simplicial complex $K_{[m]}$ and use filtration arguments to determine the homotopy type of the associated moment angle complex. We recall, $K_{[m]}$ is the simplicial complex on $[m]$ with the missing faces $\{1, \ldots, r\}$ and $\{r, r+1, \ldots, m\}$, where $r=[(m+1) / 2]$ for each integer $m \geqslant 4$. 
Lemma 6.46.

$$
\operatorname{dim} K_{[m]}= \begin{cases}2 r-3, & m \text { odd } \\ 2 r-2, & m \text { even } .\end{cases}
$$

Let $I$ be a square-free monomial ideal generated by

$$
r_{1}=v_{1} \cdots v_{r} \quad \text { and } \quad r_{2}=v_{r} v_{r+1} \cdots v_{m} .
$$

Proposition 6.47. The face ring of $K_{[m]}$ is the algebra $\mathbb{Z}\left[v_{1}, \ldots, v_{m}\right] /\langle I\rangle$.

Proof. Follows immediately from Definition 6.41 and the definition of face ring.

Recall that a simplicial complex $K$ is said to be $k$-neighborly if any set of cardinality $k-1$ is a simplex of $K$.

Lemma 6.48. For $m \geqslant 4$ an integer, $K_{[m]}$ is $(r-1)$-neighborly.

Proof. There are two cases to consider. First, we assume the cardinality of the missing faces are equal. In this case we have $m=2 k+1$ where $k \geqslant 2$. It follows that $r=k+1$. Since the missing faces consist of consecutive positive integers we have $|\{r, r+1, \ldots, m\}|=m-r+1$, which in this case is $k+1=|\{1, \ldots, r\}|$. When $m=$ $2 k, r=\left[k+\frac{1}{2}\right]=k$. In this instance, we have

$$
|\{1, . ., r\}|=k=r \quad \text { and } \quad|\{r, r+1, \ldots, m\}|=r+1 .
$$

We will use the minimal resolution of the face ring $k\left(K_{[m]}\right)$ as described in [BP4, pp. 42, 106], to determine the bi-graded Betti-numbers of the moment angle complex associated to $K_{[m]}$. As a matter of notational convenience, let $\bar{P}=\mathbb{Z}\left[v_{1}, \ldots, v_{m}\right]$ and $p \in \bar{P}$. To determine $\beta^{-i, 2 j}$ take the the minimal resolution of $k\left(K_{[m]}\right)$. This gives the following chain complex, where $x_{\Re_{\text {min }}}$ corresponds to a relation among relations, $\left|x_{\Re_{\text {min }}}\right|=\left|\Re_{\text {min }}\right|$ and the maps are defined in the obvious way:

$$
0 \stackrel{d_{4}}{\longrightarrow} R_{3} \stackrel{d_{3}}{\longrightarrow} R_{2} \stackrel{d_{2}}{\longrightarrow} R_{1} \stackrel{d_{1}}{\longrightarrow} k\left(K_{[m]}\right) \longrightarrow 0 .
$$

Here, $R_{3}=\bar{P} \otimes\left\{x_{\Re_{m i n}}\right\}, R_{2}=\bar{P} \otimes\left\{v_{1 \ldots r}, v_{r \cdot(r+1) \cdots m}\right\}$ and $R_{1}=\bar{P} \otimes\left\{\alpha_{0}\right\}$. We easily obtain

Theorem 6.49. For $m \geqslant 4$ an integer, let $Z_{K_{[m]}}$ be the associated moment angle complex. The Betti numbers of $Z_{K_{[m]}}$ are

$$
b_{k}= \begin{cases}2, & k=2 r-1 \\ 1, & k=2 m-2 \\ 0, & \text { otherwise }\end{cases}
$$

when $m$ is odd and

$$
b_{k}= \begin{cases}1, & k=2 r-1,2 m-2 r+1,2 m-2 \\ 0, & \text { otherwise }\end{cases}
$$

when $m$ is even. 
We immediately have

Corollary 6.50. For $m \geqslant 4$, an integer the moment angle complex $Z_{K_{[m]}}$ is $2 r-2$ connected.

We will now determine the homotopy type of $Z_{K_{[m]}}$. One must employ the CFSS and represent $\Re_{\text {min }}$ by the appropriate class; determine its co-action and show that the appropriate differentials are trivial. More specifically, we are particularly interested in the $d_{2}$-differential in the CFSS. We recall parts of the construction of the CFSS $[\mathbf{B C M}]$ relevant to understanding this differential. Consider the following diagram with exact rows and columns.

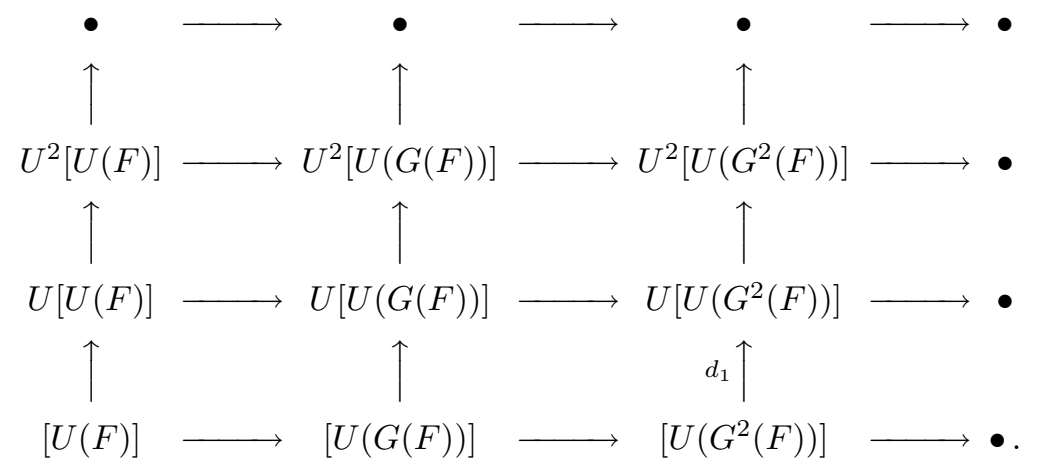

Let $x_{t} \in\left[U\left(G^{2}(F)\right)\right]$ be the $t$-dimensional class that corresponds to a cycle of $\operatorname{Ext}_{A(U)}^{0, t}\left(R^{2} P F\right) . H^{H} \cdot H^{V}$ collapses to $H_{*}(T o t)=H_{*}(F \rightarrow G(F) \cdots)$, which converges to $\operatorname{Ext}_{\mathcal{G}}^{*, *}(F)$. Hence, $H^{V} \cdot H^{H}$ is the $E_{2}$-term of a spectral sequence that converges to the $E_{2}$-term of the Novikov spectral sequence; i.e., $\operatorname{Ext}_{\mathcal{G}}^{*, *}(F) . d_{1}\left(x_{t}\right)=0$ in the vertical homology of $U\left[R^{2} P F\right]$. Hence, it is in the image of a class $\beta \in$ $U[U(G(F))]$. Push $\beta$ vertically; this is the $d_{2}$-differential that is internal to the CFSS. Note that there are differentials internal to the UANSS. One of the main goals of this section is to prove the following lemma.

Lemma 6.51. If $K$ is a simplicial complex with exactly two missing faces, then the differential

$$
\operatorname{Ext}_{A(U)}^{0,\left|\Re_{\min }\right|}\left(R^{2} P F\right) \stackrel{d-2}{\longrightarrow} \operatorname{Ext}_{A(U)}^{2,\left|\Re_{\text {min }}\right|}\left(R^{1} P F\right)
$$

is trivial.

For multi-indexes $I=\left(i_{1}, \ldots, i_{m}\right)$ and $J=\left(j_{1}, \ldots, j_{m}\right)$ consisting of zeros and ones, the pair $(I, J)$ is said to be complimentary if $I+J=\left(i_{1}+j_{1}, \ldots, i_{m}+j_{m}\right)=$ $(1, \ldots, 1)$ and if the $j^{\text {th }}$ spot in $I$ is one then the $j^{\text {th }}$ spot in $J$ must be zero. For bookkeeping purposes we introduce pairs of complimentary multi-indexes, $\left(J_{1}, J_{2}\right)$ and $\left(J_{3}, J_{4}\right)$. Consider the classes

$$
*_{j \in J_{1}} \overline{\beta_{i, j}} \circ \overline{\beta_{J_{2}}}
$$

and

$$
*_{j \in J_{3}} \overline{\beta_{i, j}} \circ \overline{\beta_{J_{4}}} .
$$

Note that the classes $\overline{\beta_{J_{2}}}$ and $\overline{\beta_{J_{2}}}$ are the unstable names for the stable classes 
$1 \otimes \beta_{J_{2}}$ and $1 \otimes \beta_{J_{4}}$. Recall that these classes came from the relations in the face ring and they did not pullback to $G(F) / U(F)$. Hence, any class divisible by these classes will not pullback to $G(F) / U(F)$ as well. In fact, this observation is essential in constructing the class that will represent the generator of $R^{2} P F$ in the $\mathrm{U}$ complex. Referring to the previous double complex we specialize to the case at hand. Let $x_{\left|\Re_{\text {min }}\right|} \in\left[U\left(G^{2}(F)\right)\right]$ be the $\left|\Re_{\text {min }}\right|$-dimensional class that corresponds to a cycle of $\operatorname{Ext}_{A(U)}^{0,\left|\Re_{\text {min }}\right|}\left(R^{2} P F\right) . d_{1}\left(x_{\left|\Re_{\text {min }}\right|}\right)=0$ in the vertical homology of $U\left[R^{2} P F\right]$. Hence, it is in the image of a class $\beta \in U[U(G(F))]$. Push $\beta$ vertically. This is the $d_{2^{-}}$ differential. We must show that $d_{2}\left(x_{\left|\Re_{\text {min }}\right|}\right)=0$. If $d_{1}\left(x_{\left|\Re_{\text {min }}\right|}\right)=0$, then $d_{2}\left(x_{\left|\Re_{\text {min }}\right|}\right)=$ 0 . Recall that the generators of $R^{1} P F$ can be represented by star products of bottomdimensional classes in $G(F)$. This was used to show that the co-action is trivial (through a range). A relation among relations is a difference in $C^{*}$ that is zero. A similar argument will be used to construct the class that represents $R^{2} P F$. Consider the following commutative diagram:

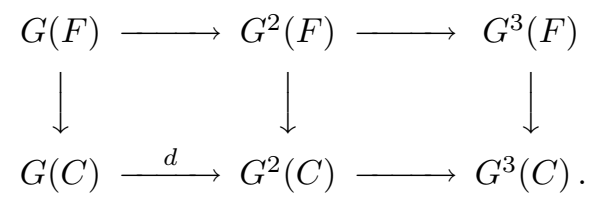

It follows immediately that $G^{i}(C)$ is a $G^{i}(F)$-module induced by the vertical maps. We need the

Lemma 6.52. $\psi: G(F) \rightarrow G^{2}(F)$ is a ring map when evaluated on the product of primitives in $G(F)$.

Proof. Consider the following commutative diagram:

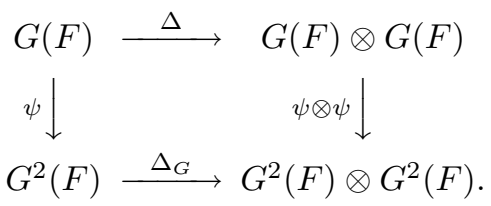

We have

$$
\begin{aligned}
\Delta_{G}(\psi(\bar{x} * \bar{y})) & =(\psi \otimes \psi)(\Delta(\bar{x} * \bar{y})) \\
& =(\psi \otimes \psi)(\Delta(\bar{x}) * \Delta(\bar{y})) .
\end{aligned}
$$

At this stage one should note that $(\Delta(\bar{x}) * \Delta(\bar{y}))$ is a bottom-dimensional class in $G(F) \otimes G(F)$ since the composition

$$
G(F) \stackrel{\Delta}{\longrightarrow} G(F) \otimes G(F) \stackrel{1 \otimes \epsilon}{\longrightarrow} G(F)
$$

is the identity. It was shown that $*$ products of bottom-dimensional classes can be identified with the completed tensor product of the same classes. We have

$$
\begin{aligned}
(\psi \otimes \psi)(\Delta(\bar{x}) * \Delta(\bar{y})) & =\psi(\Delta(\bar{x})) \otimes \psi(\Delta(\bar{y})) \\
& =\psi(\Delta(\bar{x})) * \psi(\Delta(\bar{y})) \\
& =\Delta(\psi(\bar{x})) * \Delta(\psi(\bar{y})) \\
& =\Delta(\psi(\bar{x}) * \psi(\bar{y})) .
\end{aligned}
$$


This is precisely the co-product on $G^{2}(F)$. In other words, the map $G(F) \rightarrow G^{2}(F)$ is a ring map on the primitives.

We wish to compute $d^{0}-d^{1}$ on a class that represents the generator of the second higher-derived functor of $P$ for the $B P$-face ring associated to simplicial complexes with exactly two missing faces. This map comes from taking the alternating sum of the coface maps in the cosimplicial object that arises from the $\mathcal{G}$ resolution [Bous, p. 487]. It was shown earlier that $\psi_{G(C)}\left(\beta_{J}\right)=*_{j \in J} \overline{\beta_{1, j}}+1 \otimes \beta_{J}$. The map we wish to compute is

$$
\left(d^{0}-d^{1}\right)\left(\left(*_{j \in J_{1}} \overline{\beta_{1, j}} \circ \overline{\beta_{J_{2}}}\right)-\left(*_{j \in J_{3}} \overline{\beta_{1, j}} \circ \overline{\beta_{J_{4}}}\right)\right) .
$$

Recall that $\bar{x}=b_{(0)}^{\frac{|x|}{2}}$ is the bottom-dimensional class in $G(M)$, where $M$ is a free $B P_{*}$-module on even-dimensional generators. The map $G(M) \rightarrow G^{2}(M)$ sends $\bar{x}$ to $\overline{\bar{x}}$. The copy of $B P$ associated with $\bar{x}$ is $\underline{B P}_{|x|}$, and the copy of $B P$ associated with $\overline{\bar{x}}$ is $\underline{B P} \mid \overline{x \mid}$. Since $d^{0}-d^{1}$ is linear we obtain

$$
\left(d^{0}-d^{1}\right)\left(\left(*_{j \in J_{1}} \overline{\beta_{1, j}} \circ \overline{\beta_{J_{2}}}\right)-\left(d^{0}-d^{1}\right)\left(*_{j \in J_{3}} \overline{\beta_{1, j}} \circ \overline{\beta_{J_{4}}}\right)\right) .
$$

Since $\left(d^{0}-d^{1}\right)$ is a map of Hopf rings we obtain

$$
\begin{aligned}
d^{0}\left(\left(*_{j \in J_{1}} \overline{\beta_{1, j}} \circ \overline{\beta_{J_{2}}}\right)\right)-d^{1}\left(\left(*_{j \in J_{1}} \overline{\beta_{1, j}} \circ \overline{\beta_{J_{2}}}\right)\right) \\
\\
-d^{0}\left(\left(*_{j \in J_{3}} \overline{\beta_{1, j}} \circ \overline{\beta_{J_{4}}}\right)\right)-d^{1}\left(\left(*_{j \in J_{3}} \overline{\beta_{1, j}} \circ \overline{\beta_{J_{4}}}\right)\right) .
\end{aligned}
$$

Recall that from the triple structure we have $d^{0}=\eta \circ G$ and $d^{1}=G \circ \eta$.

We obtain

$$
\begin{aligned}
d^{0}\left(\left(*_{j \in J_{1}} \overline{\beta_{1, j}}\right) \circ d^{0}\left(\overline{\beta_{J_{2}}}\right)\right. & -d^{1}\left(\left(*_{j \in J_{1}} \overline{\beta_{1, j}}\right)\right) \circ d^{1}\left(\overline{\beta_{J_{2}}}\right) \\
& -\left[d^{0}\left(\left(*_{j \in J_{3}} \overline{\beta_{1, j}}\right) \circ d^{0}\left(\overline{\beta_{J_{4}}}\right)\right]-d^{1}\left(\left(*_{j \in J_{3}} \overline{\beta_{1, j}}\right)\right) \circ d^{1}\left(\overline{\beta_{J_{4}}}\right)\right) .
\end{aligned}
$$

To compute the map $d^{1}\left(\overline{\beta_{J}}\right)$, we compute the unstable co-algebra map $\psi_{G(C)}$ on the $\beta$ whose image under this map is the stable part of the class in $G(C)$. In addition to this observation, we use the map $G(M) \rightarrow G^{2}(M)$ and the Hopf ring relation $a \circ(b * c)=(a \circ b) *(a \circ c)$ to obtain the class

$$
*_{j \in J_{3}} \overline{\overline{\beta_{1, j}}} \circ *_{j \in J_{4}} \overline{\overline{\beta_{1, j}}}-*_{j \in J_{1}} \overline{\overline{\beta_{1, j}}} \cdot \circ *_{j \in J_{2}} \overline{\overline{\beta_{1, j}}} .
$$

Call this class $\Re_{\min }$. It is important to note that the notation can be misleading. The following example should be illuminating.

Example 6.53. Let $K$ be a simplicial complex on the vertex set [5] with missing faces $\{1,2,3\}$ and $\{3,4,5\}$. Clearly, the relation among relations in this example is $\iota^{*}\left(x_{1}\right) v_{4} v_{5}-\iota^{*}\left(x_{2}\right) v_{1} v_{2}$. Let $x_{10} \in\left[U\left(G^{2}(F)\right)\right]$ be the ten-dimensional class that corresponds to a cycle of $\operatorname{Ext}_{A(U)}^{0,10}\left(R^{2} P F\right)$. We want to show that the differential

$$
\operatorname{Ext}_{A(U)}^{0,10}\left(R^{2} P F\right) \stackrel{d_{2}}{\longrightarrow} \operatorname{Ext}_{A(U)}^{2,10}\left(R^{1} P F\right)
$$

is trivial. In this example, $\overline{\beta_{(1,1,1,0,0)}}$ is the unstable name for the stable class 
$1 \otimes \beta_{(1,1,1,0,0)}$. Consider the class:

$$
\left(\overline{\beta_{1,4}} * \overline{\beta_{1,5}}\right) \circ \overline{\beta_{(1,1,1,0,0)}} .
$$

Similarly, we have the class

$$
\left(\overline{\beta_{1,1}} * \overline{\beta_{1,2}}\right) \circ \overline{\beta_{(0,0,1,1,1)}} .
$$

We must compute $\left.\left(d^{0}-d^{1}\right)\left(\overline{\beta_{1,4}} * \overline{\beta_{1,5}}\right) \circ \overline{\beta_{(1,1,1,0,0)}}-\left(\overline{\beta_{1,1}} * \overline{\beta_{1,2}}\right) \circ \overline{\beta_{(0,0,1,1,1)}}\right)$.

To illustrate the computation we will compute $\left.\left(d^{0}-d^{1}\right)\left(\overline{\beta_{1,4}} * \overline{\beta_{1,5}}\right) \circ \overline{\beta_{(1,1,1,0,0)}}\right)$.

$$
\begin{aligned}
\left(d^{0}-d^{1}\right) & \left.\left(\overline{\beta_{1,4}} * \overline{\beta_{1,5}}\right) \circ \overline{\beta_{(1,1,1,0,0)}}\right) \\
& \left.\left.=d^{0}\left(\overline{\overline{\beta_{1,4}}} * \overline{\beta_{1,5}}\right) \circ \overline{\beta_{(1,1,1,0,0)}}\right)-d^{1}\left(\overline{\overline{\beta_{1,4}}} * \overline{\beta_{1,5}}\right) \circ \overline{\beta_{(1,1,1,0,0)}}\right) \\
& \left.\left.\left.=\overline{\overline{\left(\beta_{1,4}\right.}} * \overline{\overline{\beta_{1,5}}}\right) \circ \overline{\overline{\beta_{(1,1,1,0,0)}}}\right)-d^{1}\left(\overline{\beta_{1,4}} * \overline{\beta_{1,5}}\right) \circ \overline{\beta_{(1,1,1,0,0)}}\right) \\
& \left.\left.\left.=\overline{\overline{\left(\beta_{1,4}\right.}} * \overline{\overline{\beta_{1,5}}}\right) \circ \overline{\overline{\beta_{(1,1,1,0,0)}}}\right)-d^{1}\left(\overline{\beta_{1,4}} * \overline{\beta_{1,5}}\right)\right) \circ d^{1}\left(\overline{\beta_{(1,1,1,0,0)}}\right) \\
& \left.\left.=\overline{\overline{\left(\beta_{1,4}\right.}} * \overline{\overline{\beta_{1,5}}}\right) \circ \overline{\overline{\beta_{(1,1,1,0,0)}}}\right)-\left(\overline{\overline{\beta_{1,4}}} * \overline{\overline{\beta_{1,5}}}\right) \circ d^{1}\left(\overline{\beta_{(1,1,1,0,0)}}\right) .
\end{aligned}
$$

To compute $d^{1}\left(\overline{\beta_{(1,1,1,0,0)}}\right)$ We compute $\psi_{G(C)}$ on the $\beta$ whose image gives the stable part of the class in $G(C)$ whose unstable name is $\left(\overline{\beta_{(1,1,1,0,0)}}\right)$. Then we push into $G^{2}(C)$. Therefore,

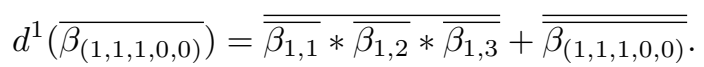

Combining this with the computation above we have

$$
\begin{aligned}
&\left.\left(d^{0}-d^{1}\right)\left(\overline{\beta_{1,4}} * \overline{\beta_{1,5}}\right) \circ \overline{\beta_{(1,1,1,0,0)}}\right) \\
&\left.\left.\quad=\overline{\left(\overline{\beta_{1,4}}\right.} * \overline{\overline{\beta_{1,5}}}\right) \circ \overline{\overline{\beta_{(1,1,1,0,0)}}}\right)-\left(\overline{\overline{\beta_{1,4}}} * \overline{\overline{\beta_{1,5}}}\right) \circ\left(\overline{\overline{\beta_{1,1}}} * \overline{\beta_{1,2}} * \overline{\beta_{1,3}}+\overline{\overline{\left.\beta_{(1,1,1,0,0}\right)}}\right) .
\end{aligned}
$$

Distributing and canceling gives

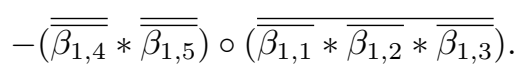

Similarly, for the second class, we have, after distributing and cancellation the class

$$
-\left(\overline{\overline{\beta_{1,1}}} * \overline{\overline{\beta_{1,2}}}\right) \circ\left(\overline{\overline{\beta_{1,3}} * \overline{\beta_{1,4}} * \overline{\beta_{1,5}}}\right) .
$$

Using the linearity of $d^{0}-d^{1}$ along with the Hopf ring relation described earlier, we obtain the class

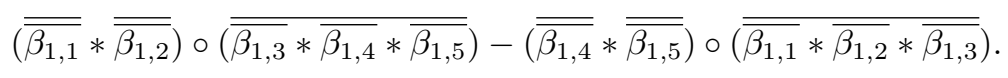

This class represents the generator of $R^{2} P F$.

Now we give the proof of Lemma 6.51. 
Proof. Consider the following commutative diagram:

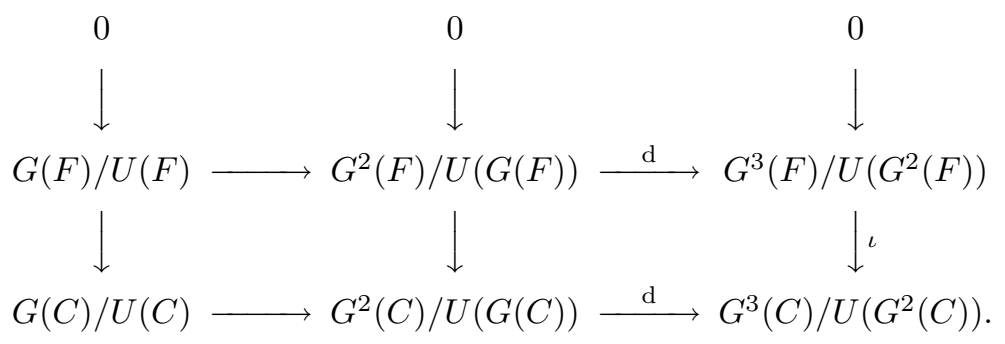

Since $\left.d\left(d\left(\Re_{\min }\right)\right)\right)=0$, the class $d\left(\Re_{\min }\right)$ pulls back to $G^{2}(F)$. If we start with the class $\Re_{\text {min }}$, then it follows from the observations above that $d\left(\Re_{\text {min }}\right)$ is a cycle in this complex representing a class in $R^{2} P F$ that cannot be pulled back to $G(F) / U(F)$. It follows immediately from this diagram that the map $R^{2} P F \rightarrow U\left(R^{2} P F\right)$ is zero. Hence, $d_{1}\left(x_{\left|\Re_{\text {min }}\right|}\right)=0$. Therefore, the $d_{2}$-differential above is zero.

Theorem 6.54. Let $m \geqslant 4$ be an integer and $Z_{K_{[m]}}$ the associated moment angle complex. We have

$$
Z_{K_{[m]}} \simeq \begin{cases}S^{2 r-1} \bigvee S^{2 m-2 r+1} \bigvee S^{2 m-2}, & m \text { even } \\ S^{2 r-1} \bigvee S^{2 r-1} \bigvee S^{2 m-2}, & m \text { odd }\end{cases}
$$

Proof. The dimension of the top-dimensional cell is $2 m-2$ independent of the parity of $m$. Suppose $f: S^{2 m-3} \rightarrow S^{i} \bigvee S^{j}$ is a non-trivial attaching map, where $i=j=2 r-1$ if $m$ is odd and $i=2 r-1, j=2 m-2 r+1$ otherwise. Since $f$ is nontrivial, the smallest possible differential in the UANSS is a $d_{2}$-differential out of $(2,2 m)$ into $(4,2 m+1)$. The classes $\eta^{i} \cdot \iota$ for $i \leqslant 3$ are even-dimensional. Hence, the smallest possible differential is a $d_{3}$ which jumps to filtration 5 . Therefore, all the classes survive. Hence, $f$ is trivial. The same argument works for $m$ even. The only possible differential internal to the CFSS that can possibly kill classes that may survive to the $E_{2}$-term of the UANSS is the $d_{2}$ described earlier. The proof follows by Lemma 6.51.

$K_{[m]}$ is not shifted. This can been seen by observing that the missing faces of this simplicial complex are $\{1, \ldots, r\}$ and $\{r, r+1, \ldots, m\}$. Let

$$
\sigma=\{1, \ldots, r-1, r+1, r+2, \ldots, m\} .
$$

It follows from the definition of $K_{[m]}$ that

$$
\sigma \backslash\{r+1\}=\{1, \ldots, r-1, r+2, \ldots, m\} \in K_{[m]} .
$$

However, $\sigma \backslash\{r+1\} \cup\{r\} \notin K_{[m]}$.

Proposition 6.55. $k\left(K_{[m]}\right)$ is a Golod ring.

Proof. By Theorem 6.54, $Z_{K_{[m]}}$ is a wedge of spheres. The result follows from [GT2, Theorem 1.5].

In [GT2] the homotopy type of $Z_{K}$ is determined when $K$ is a shifted complex. 
Miscellaneous results on the homotopy groups of certain quasi-toric manifolds, connected sums and wedges via toric geometry

In this section we gather some miscellaneous results on the homotopy groups of $M^{2 n}(\lambda)$ that are probably known. However, they do not appear in the literature. The following proposition is enormously important and was communicated by A. Bahri. We assume that $P$ is a $n$-dimensional simple convex polytope and that $M^{2 n}(\lambda)$ is the family of quasi-toric manifolds that sit over $P$. Even though quasi-toric manifolds depend on the choice of $\lambda$ their homotopy groups do not as illustrated by

Proposition 6.56. Let $M^{2 n}\left(\lambda_{1}\right)$ and $M^{2 n}\left(\lambda_{2}\right)$ be two quasi-toric manifolds on the same underlying polytope, then $\pi_{*}\left(M^{2 n}\left(\lambda_{1}\right)\right) \cong \pi_{*}\left(M^{2 n}\left(\lambda_{2}\right)\right)$ for $* \geqslant 3$.

Proof. Let $\lambda_{1}$ and $\lambda_{2}$ be two maps from $\mathbb{Z}^{m} \rightarrow \mathbb{Z}^{n}$ satisfying condition $(*)$ such that $\lambda_{1} \neq \lambda_{2}$. Let $M^{2 n}\left(\lambda_{1}\right)$ and $M^{2 n}\left(\lambda_{2}\right)$ be the corresponding quasi-toric manifolds. Using the fibration

$$
M^{2 n} \rightarrow B_{T} P \rightarrow \prod_{n} C P^{\infty}
$$

we obtain

$$
M^{2 n}\left(\lambda_{1}\right) \rightarrow B_{T} P \rightarrow \prod_{n} C P^{\infty}
$$

and

$$
M^{2 n}\left(\lambda_{2}\right) \rightarrow B_{T} P \rightarrow \prod_{n} C P^{\infty}
$$

The Borel space $B_{T} P$ is fixed for each $\lambda$ and only depends on $P$. Apply $\pi_{*}(-)$ and the result follows.

In other words the higher homotopy groups of a quasi-toric manifold are independent of $\lambda$.

$B_{n}$, first introduced in [BR1], is the quasi-toric manifold of all bounded flags in $\mathbb{C}^{n+1}[\mathbf{B R 2}]$. Its quotient polytope is the cube $I^{n}$.

Proposition 6.57. For $* \geqslant 3, \pi_{*}\left(B_{n}\right) \cong \pi_{*}\left(\prod_{n} S^{3}\right)$.

Proof. $I^{n}$ is combinatorially equivalent to the simple convex polytope $\prod_{n} \Delta^{1}$. By [BP1], $Z_{\prod_{n} \Delta^{1}} \cong \prod_{n} S^{3}$. The result follows by applying $\pi_{*}(-)$ to the fibration:

$$
T^{m-n} \rightarrow Z_{P} \rightarrow M^{2 n}
$$

Recall from $[\mathbf{B R 2}]$ and $[\mathbf{B R 3}] B_{i, j}$-the family of quasi-toric manifolds that sit over $I^{i} \times \Delta^{j-1}$. This leads to our next observation:

Proposition 6.58. For $* \geqslant 3, \pi_{*}\left(B_{i, j}\right) \cong \pi_{*}\left(\prod_{i} S^{3} \times S^{2 j-1}\right)$.

Proof. Apply the argument given in the proof of Proposition 6.57.

There is no known technique that allows for the computation of $\pi_{*}\left(\bigvee_{m} \mathbb{C} P^{\infty}\right)$. It will be shown that the homotopy of $\bigvee_{m} \mathbb{C} P^{\infty}$ is given by the homotopy of a particular wedge of spheres. Of course, the Milnor-Hilton theorem can be used to make homotopy computations. 
Theorem 6.59. For $* \geqslant 3, \pi_{*}\left(C P^{n} \sharp C P^{n}\right) \cong \pi_{*}\left(S^{3}\right) \oplus \pi_{*}\left(S^{2 n-1}\right)$.

Proof. [BR2] proved that $\mathbb{C} P^{n} \sharp \mathbb{C} P^{n}$ is a quasi-toric manifold over $\Delta^{n} \sharp \Delta^{n}$. This polytope is combinatorially equivalent to the simple convex polytope $\Delta^{1} \times \Delta^{n-1}[\mathbf{B P 4}]$. The result follows by applying $\pi_{*}(-)$ to the fibration $T^{m-n} \rightarrow Z_{P} \rightarrow M^{2 n}$ and observing that the moment angle complex behaves well with respect to products.

Lemma 6.60. If $K$ is the disjoint union of $m$ vertices, then

$$
D J(K)=\bigvee_{m} \mathbb{C} P^{\infty} .
$$

Proof. Using the Strickland construction $D J(K)=K_{\bullet}\left(\mathbb{C} P^{\infty}\right)$, one computes

$$
K_{\bullet}\left(\mathbb{C} P^{\infty}, *\right)=\bigcup_{\sigma \in K}\left(\prod_{i \in \sigma} \mathbb{C} P^{\infty} \times \prod_{i \notin \sigma}\{*\}\right) .
$$

More explicitly we have

$$
K_{\bullet}\left(\mathbb{C} P^{\infty}, *\right)=\mathbb{C} P^{\infty} \times\{*\} \times \cdots \times\{*\} \bigcup \cdots \bigcup\{*\} \times \cdots \times\{*\} \times \mathbb{C} P^{\infty}
$$

with the obvious identifications along the boundary.

When $K$ is the disjoint union of $m$, vertices $[\mathbf{G T}]$ shows that $U(K)$ is a wedge of spheres. This leads to the following

Theorem 6.61. For $* \geqslant 3, \pi_{*}\left(\bigvee_{m} \mathbb{C} P^{\infty}\right) \cong \pi_{*}\left(\bigvee_{k=2}^{n}(k-1)\left(\begin{array}{l}n \\ k\end{array}\right) S^{k+1}\right)$.

Proof. The result follows from Lemma 6.60 combined with the fact that $D J(K)$ is a deformation retract of $B_{T} Z_{K}$ and $U(K)$ is a deformation retract of $Z_{K}$.

\section{References}

[A] J.F. Adams, Stable homotopy and generalized homology, University of Chicago Press, Chicago, IL, 1974.

[Bahri] A. Bahri, private communication.

[Bend] M. Bendersky, The derived functors of the primitives for $B P_{*}\left(\Omega S^{2 n+1}\right)$, Trans. Amer. Math. Soc. 276 (1983), 599-619.

[Bend2] M. Bendersky, The $v_{1}$-periodic unstable Novikov spectral sequence, Topology 33, no. 1 (1992), 47-64.

[BCM] M. Bendersky, E.B. Curtis, and H.R. Miller, The unstable Adams spectral sequence for generalized homology, Topology 17 (1978), 229-248.

[BCR] M. Bendersky, E.B. Curtis, and D.C. Ravenel, EHP sequences in BP theory, Topology 21, no. 4 (1982), 373-391.

[BDM] M. Bendersky, D.M. Davis, and M. Mahowald, vi-periodic homotopy groups of $\mathrm{SP}(n)$, Pacific J. Math. 170, no. 2 (1995), 319-378.

[BH] M. Bendersky and J. Hunton, On the co-algebraic ring and Bousfield-Kan spectral sequence for a Landweber exact spectrum, Proc. Edinburgh Math. Soc. 47, no. 3 (2004), 513-532. 
[BT] M. Bendersky and R. Thompson, The Bousfield-Kan spectral sequence for periodic homology theories, Amer. J. Math. 122, no. 3 (2000), 599-635.

[Bous] A.K. Bousfield, Nice homology co-algebras, Trans. Amer. Math. Soc. 148 (1970), 473-489.

[Bron] A. Brønsted, An introduction to convex polytopes, Grad. Texts in Math. 90, Springer-Verlag, New York, 1983.

[BP1] V.M. Buchstaber and T.E. Panov, Torus actions and the combinatorics of polytopes, Proceedings of the Stekolv Institute of Mathematics 225 (1999), $87-120$.

[BP2] V.M. Buchstaber and T.E. Panov, Torus actions, combinatorial topology and homological algebra, Russian Math. Surveys 55 (2000), no. 5, 825-921.

[BP3] V.M. Buchstaber and T.E. Panov, Torus actions, equivariant momentangle complexes, and coordinate subspace arrangements, J. Math. Sciences 113, no. 4 (2003), 558-568.

[BP4] V.M. Buchstaber and T.E. Panov, Torus actions and their applications in topology and combinatorics, University Lecture Series 24, A. M. S., Providence, RI (2002).

[BR1] V.M. Buchstaber and N. Ray, Flag manifolds and the Landweber-Novikov algebra, Geometry and Topology 2 (1998), 79-101 (electronic).

[BR2] V.M. Buchstaber and N. Ray, Tangential structures on toric manifolds, and connected sums of polytopes, Internat. Math. Res. Notices 2001, no. 4, 193-219.

[BR3] V.M. Buchstaber and N. Ray, Toric manifolds and complex cobordism, Russian Math. Surveys 53, no. 2 (1998), 371-373.

[Civ] Y. Civan, Examples from toric geometry, preprint; math. AT/0306029.

[Dan] V. Danilov, The geometry of toric varieties, Uspekhi Mat. Nauk 33 (1978), no. 2, 85-134; English translation in Russian Math. Surveys 33 (1978), no. $2,97-154$.

[DP] A. Dimca and S. Papadima, Hypersurface complements, Milnor fibers and higher homotopy groups of arrangements, Annals of Math. 158 no. 2 (2003), 473-507.

[Dob] N. E. Dobrinskaya, The classification problem for quasi-toric manifolds over a given simple polytope, Funct. Anal. Appl. 35, no. 2 (2001), 83-89.

[DJ] M. Davis and T. Januszkiewicz, Convex polytopes, Coxeter orbifolds and torus actions, Duke Math. J. 62, no. 2 (1991), 417-451.

[GT] J. Grbic and S. Theriault, The homotopy type of the complement of the codimension two coordinate subspace arrangement, Uspekhi Mat. Nauk 59, no. 6, 203-204; English translation in Russian Math. Surveys 59, no. 3 (2004), 1207-1209.

[GT2] J. Grbic and S. Theriault, The homotopy type of the complement of a coordinate subspace arrangement, Topology 46, no. 4 (2007), 357-396. 
[L] E. Lerman, Homotopy groups of $K$-contact toric manifolds, Trans. Amer. Math. Soc. 356, no. 10 (2004), 4075-4083.

[MS] J.C. Moore and L. Smith, Hopf algebras and multiplicative fibrations II, Amer. J. Math. 90 (1968), 1113-1150.

[OR] P. Orlik and F. Raymond, Actions of the torus on 3-manifolds I, Trans. Amer. Math. Soc. 152 (1970), 531-559.

[PS] S. Papadima and A. Suciu, Higher homotopy groups of complements of complex hyperplane arrangements, Adv. in Math. 165 (2002), 209-223.

[RW] D.C. Ravenel and W.S. Wilson, The Hopf ring for complex cobordism, $J$. Pure Applied Algebra 9 (1977), 241-280.

[S] R. Switzer, Algebraic topology-homology and homotopy, Springer-Verlag, New York, 1975.

[W] C. Weibel, An introduction to homological algebra, Cambridge University Press, Cambridge, 1994.

[Zie] G. Ziegler, Homotopy types of subspace arrangements via diagrams of spaces, Math. Ann. 295 (1993), 527-548.

David Allen dallen@iona.edu

Department of Mathematics, Iona College, New Rochelle, NY, 10801, USA 